\title{
Tetraalkoxyphenanthrene: A New Precursor for Luminescent Conjugated Polymers
}

\author{
Britta N. Boden, Katherine J. Jardine, Alfred C. W. Leung, Mark J. MacLachlan*
}

Department of Chemistry, University of British Columbia, 2036 Main Mall, Vancouver, BC. V6T 1Z1

mmaclach@chem.ubc.ca

\section{Supplementary Material}




\section{Table of Contents}

$\begin{array}{ll}\text { Experimental Methods and Characterization } & \text { S2 }\end{array}$

Figure 1. ${ }^{1} \mathrm{H}$ NMR spectrum of $3 . \quad$ S9

Figure 2. ${ }^{13} \mathrm{C}$ NMR spectrum of $3 . \quad$ S9

Figure 3. ${ }^{1} \mathrm{H}$ NMR spectrum of $4 . \quad$ S10

Figure 4. ${ }^{13} \mathrm{C}$ NMR spectrum of $4 . \quad \mathrm{S} 10$

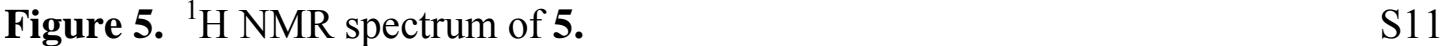

Figure 6. ${ }^{13} \mathrm{C}$ NMR spectrum of 5 . $\quad$ S11

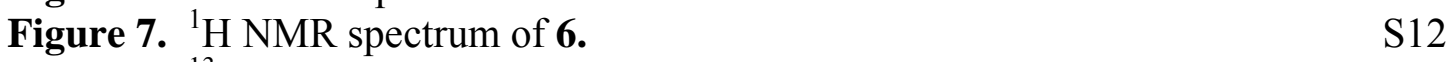

$\begin{array}{lr}\text { Figure 8. } & { }^{13} \mathrm{C} \text { NMR spectrum of } 6 . \\ \text { S12 }\end{array}$

$\begin{array}{ll}\text { Figure 9. }{ }^{1} \mathrm{H} \text { NMR spectrum of 7a. } & \text { S13 }\end{array}$

$\begin{array}{ll}\text { Figure 10. }{ }^{13} \mathrm{C} \text { NMR spectrum of 7a. } & \text { S13 }\end{array}$

$\begin{array}{ll}\text { Figure 11. }{ }^{1} \mathrm{H} \text { NMR spectrum of } \mathbf{7 b} . & \mathrm{S} 14\end{array}$

Figure 12. ${ }^{13} \mathrm{C}$ NMR spectrum of $\mathbf{7 b} . \quad \mathrm{S} 14$

Figure 13. ${ }^{1} \mathrm{H}$ NMR spectrum of 7c. $\quad$ S15

$\begin{array}{ll}\text { Figure 14. }{ }^{13} \mathrm{C} \text { NMR spectrum of 7c. } & \text { S15 }\end{array}$

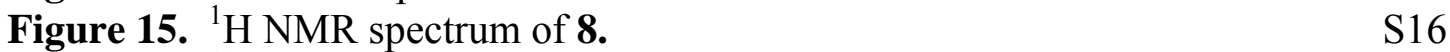

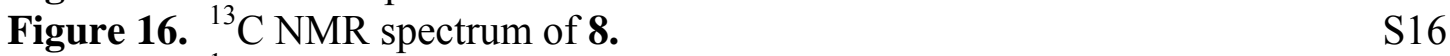

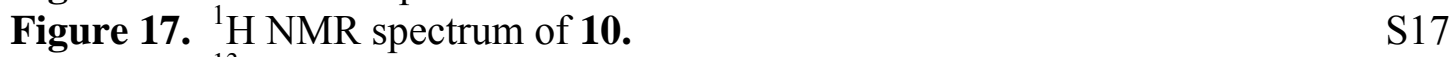

$\begin{array}{lr}\text { Figure 18. }{ }^{13} \mathrm{C} \text { NMR spectrum of 10. } & \text { S17 }\end{array}$

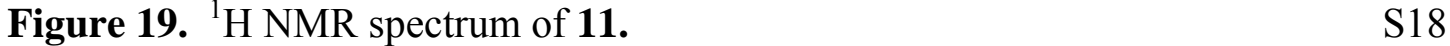

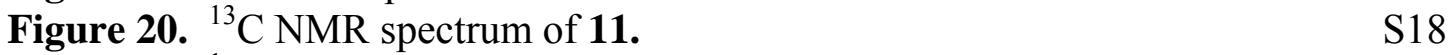

$\begin{array}{lr}\text { Figure 21. }{ }^{1} \mathrm{H} \text { NMR spectrum of 13. } & \text { S19 }\end{array}$

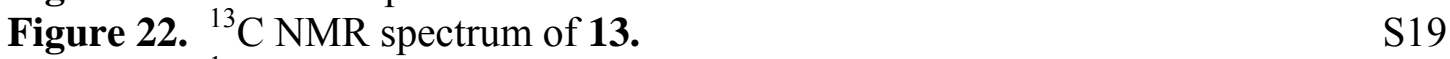

Figure 23. ${ }^{1} \mathrm{H}$ NMR spectrum of $\mathbf{1 4} . \quad$ S20

Figure 24. ${ }^{13} \mathrm{C}$ NMR spectrum of $14 . \quad$ S20

\section{Experimental Section}

Materials and Equipment. Tetrahydrofuran (THF) was distilled over $\mathrm{Na}$ and benzophenone under $\mathrm{N}_{2}$. Diisopropylamine was distilled from $\mathrm{NaOH}$ under $\mathrm{N}_{2}$. 3,6Dibromo-9,10-phenanthrenequinone $2,{ }^{1}$ 1,4-diethynyl-2,5-dihexadecyloxybenzene $\mathbf{9}^{2}$ and 1,4-divinyl-2,5-dihexadecyloxybenzene $\mathbf{1 2}^{3}$ were prepared according to literature procedures. Bis(triphenylphosphine)palladium(II) dichloride and tetrakis(triphenylphosphine)palladium(0) were obtained from Strem Chemicals, Inc. Deuterated solvents were obtained from Cambridge Isotope Laboratories, Inc. All other chemicals were purchased from Aldrich or Fisher and used as received. All reactions were carried out under nitrogen unless otherwise noted. ${ }^{1} \mathrm{H}$ NMR (300 or $400 \mathrm{MHz}$ ) and ${ }^{13} \mathrm{C}$ NMR (75.5 or $100.6 \mathrm{MHz}$ ) spectra were recorded on Bruker Avance 300 or Bruker Avance 400 spectrometers and were referenced internally to residual protonated solvent. Infrared spectra were obtained as $\mathrm{KBr}$ discs or on $\mathrm{NaCl}$ plates with a Bomem MB-100 spectrometer. UV-Vis spectra were obtained in HPLC grade $\mathrm{CH}_{2} \mathrm{Cl}_{2}$ on a Varian Cary $5000 \mathrm{UV}-\mathrm{Vis} /$ near IR spectrometer using a $1 \mathrm{~cm}$ cuvette or a microscope slide. Fluorescence spectra were obtained in $\mathrm{CH}_{2} \mathrm{Cl}_{2}$ on a PTI QuantaMaster fluorimeter using a 
$1 \mathrm{~cm}$ quartz cuvette or a microscope slide. Quantum yields were referenced to a solution of anthracene in $\operatorname{EtOH}(\Phi=0.30){ }^{4}$ Electron ionization $(\mathrm{EI})$ and electrospray ionization (ESI) mass spectra were obtained in the UBC Mass Spectrometry facility. Elemental analyses were obtained at the UBC Microanalytical facility. Melting points were obtained on a Fisher John's melting point apparatus. Molecular weights were estimated by gel permeation chromatography (GPC) using a Waters liquid chromatograph equipped with a Waters 515 HPLC pump, Waters 717 plus autosampler, Waters Styragel $\mathbb{R}$ columns (4.6\% $300 \mathrm{~mm}) \mathrm{HR} 2, \mathrm{HR} 4$ and HR5E and a Waters 2410 differential refractometer (refractive index detector). A flow rate of $0.3 \mathrm{~mL} / \mathrm{min}$ was used and samples were dissolved in THF (ca. $1 \mathrm{mg} / \mathrm{mL}$ ) and filtered before injection. Narrow molecular weight polystyrene standards were used for calibration purposes. It is wellknown that GPC analysis of rigid-rod polymers can overestimate the molecular weights of polymers by as much as $50 \%$ when polystyrene standards are employed.

Synthesis of 3,6-dibromo-9,10-dimethoxyphenanthrene (3). Compound 3 was prepared using a published procedure for related compounds. ${ }^{5}$ Compound 2 (11.12 g, $30.3 \mathrm{mmol}), \mathrm{Bu}_{4} \mathrm{NBr}(2.85 \mathrm{~g}, 8.82 \mathrm{mmol}), \mathrm{Na}_{2} \mathrm{~S}_{2} \mathrm{O}_{4}(16.018 \mathrm{~g}, 91.9 \mathrm{mmol})$, THF (100 mL) and $\mathrm{H}_{2} \mathrm{O}(100 \mathrm{~mL})$ were combined in a separatory funnel and shaken for $5 \mathrm{~min}$, after which dimethyl sulfate $(15 \mathrm{~mL}, 159 \mathrm{mmol})$ was added, followed by aqueous sodium hydroxide $(30 \mathrm{~mL}, 14.1 \mathrm{M})$. The mixture was shaken for $3 \mathrm{~min}$, during which, $100 \mathrm{~g}$ of ice was added, then the mixture was shaken for 12 additional minutes. The aqueous layer was separated and extracted with ethyl acetate (EtOAc) $(3 \times 150 \mathrm{~mL})$. The combined organic layers were washed with water $(3 \times 100 \mathrm{~mL}), \mathrm{NH}_{4} \mathrm{OH}$ solution $(2 \times 100 \mathrm{~mL})$ and brine $(1 \times 100 \mathrm{~mL})$. The organic layer was dried with $\mathrm{MgSO}_{4}$, filtered and the solvents were removed under vacuum, resulting in a fluffy yellow solid. Washing the product with methanol $(\mathrm{MeOH})$ gave a white solid. Additional impurities were removed by flashing the product through silica with a 1:1 mixture of hexanes and methylene chloride (DCM). Yield: $10.86 \mathrm{~g}, 27.4 \mathrm{mmol}, 90 \%$

Data for 3. ${ }^{1} \mathrm{H}$ NMR $\left(400 \mathrm{MHz}, \mathrm{CDCl}_{3}\right) \delta 8.63(\mathrm{~d}, 2 \mathrm{H}, J=1.8 \mathrm{~Hz}$, aromatic $\mathrm{CH}), 8.07$ (d, $2 \mathrm{H}, J=8.8 \mathrm{~Hz}$, aromatic $\mathrm{CH}), 7.70\left(\mathrm{dd}, 2 \mathrm{H}, J_{1}=8.8 \mathrm{~Hz}, J_{2}=1.8 \mathrm{~Hz}\right.$, aromatic $\left.\mathrm{CH}\right), 4.05$ $\left(\mathrm{s}, 6 \mathrm{H}, \mathrm{OCH}_{3}\right) ;{ }^{13} \mathrm{C} \mathrm{NMR}\left(75.5 \mathrm{MHz}, \mathrm{CDCl}_{3}\right) \delta 144.0,130.8,129.1,128.4,125.6,124.2$, 120.7, 61.2; ESI-MS: $m / z=419\left([\mathrm{M}+\mathrm{Na}]^{+}\right)$; IR $(\mathrm{KBr}): v=2964,2934,1619,1589,1483$, $1424,1347,1313,1121,1094,1066,981,868,813 \mathrm{~cm}^{-1}$; UV-Vis $\left(\mathrm{CH}_{2} \mathrm{Cl}_{2}\right) \lambda_{\max }(\varepsilon)=253$ $\left(4.9 \times 10^{4}\right), 260\left(5.4 \times 10^{4}\right), 282\left(1.8 \times 10^{4}\right), 302\left(1.3 \times 10^{4}\right), 315\left(1.4 \times 10^{4}\right) \mathrm{nm}\left(\mathrm{L} \mathrm{mol}^{-1}\right.$ $\left.\mathrm{cm}^{-1}\right)$; Mp. $=162-163{ }^{\circ} \mathrm{C}$; Anal. Calc'd for $\mathrm{C}_{16} \mathrm{H}_{12} \mathrm{O}_{2} \mathrm{Br}_{2}: \mathrm{C}, 48.52, \mathrm{H}, 3.05$. Found $\mathrm{C}$, 48.39, H, 3.21.

Synthesis of 3,6,9,10-tetramethoxyphenanthrene (4). EtOAc (1 mL) and toluene (1 $\mathrm{mL})$ were added to $3(2.26 \mathrm{~g}, 5.71 \mathrm{mmol})$, under $\mathrm{N}_{2}$. To this mixture was added $\mathrm{NaOMe} / \mathrm{MeOH}(25 \mathrm{wt} \%, 50 \mathrm{~mL})$ and $\mathrm{CuBr}(0.081 \mathrm{~g}, 0.571 \mathrm{mmol})$. The mixture was heated to $80{ }^{\circ} \mathrm{C}$ for $16 \mathrm{~h}$. After cooling to room temperature, the solution was poured into $150 \mathrm{~mL}$ water and extracted with DCM (3 x $150 \mathrm{~mL})$, dried over $\mathrm{MgSO}_{4}$, and filtered. Rotary evaporation of the solution gave a brown oil which was passed through silica in 1:1 hexanes to DCM. Recrystallization of the product from ethanol (EtOH) afforded 1.66 $\mathrm{g}(5.58 \mathrm{mmol}, 98 \%)$ of a white solid. 
Data for 4. ${ }^{1} \mathrm{H}$ NMR $\left(300 \mathrm{MHz}, \mathrm{CDCl}_{3}\right) \delta 8.11$ (d, $2 \mathrm{H}, J=9.0 \mathrm{~Hz}$, aromatic), 7.89 (d, $2 \mathrm{H}, J=2.5 \mathrm{~Hz}$ aromatic), $7.25\left(\mathrm{dd}, 2 \mathrm{H}, J_{1}=9.0 \mathrm{~Hz}, J_{2}=2.5 \mathrm{~Hz}\right.$, aromatic), $4.04(\mathrm{~s}, 6 \mathrm{H}$, $\left.\mathrm{OCH}_{3}\right), 3.99\left(\mathrm{~s}, 6 \mathrm{H}, \mathrm{OCH}_{3}\right) ;{ }^{13} \mathrm{C}$ NMR $\left(75.5 \mathrm{MHz}, \mathrm{CDCl}_{3}\right) \delta 157.9,142.5,129.5,124.2$, 124.0, 116.7, 104.9, 61.2, 55.8; ESI-MS: $m / z=321\left([\mathrm{M}+\mathrm{Na}]^{+}\right)$; IR $(\mathrm{KBr}): v=2996$, 2960, 2936, 2835, 1603, 1508, 1450, 1430, 1354, 1319, 1260, 1233, 1172, 1122, 1068, 1029, 986, 849, $832 \mathrm{~cm}^{-1}$; UV-Vis $\left(\mathrm{CH}_{2} \mathrm{Cl}_{2}\right) \lambda_{\max }(\varepsilon)=252\left(4.1 \times 10^{4}\right), 291\left(1.7 \times 10^{4}\right)$, $313\left(1.1 \times 10^{4}\right) \mathrm{nm}\left(\mathrm{L} \mathrm{mol}^{-1} \mathrm{~cm}^{-1}\right)$; Mp. = 74-76 ${ }^{\circ} \mathrm{C}$; Anal. Calc'd for $\mathrm{C}_{18} \mathrm{H}_{18} \mathrm{O}_{4}$ : C, 72.47; H, 6.08. Found: C, 72.18; H, 6.12.

Synthesis of 3,6-dimethoxyphenanthrene-9,10-quinone (5). Compound 4 (1.017 g, 3.4 mmol) was dissolved in acetonitrile $(20 \mathrm{~mL})$. A solution of ammonium cerium(IV) nitrate $(3.73 \mathrm{~g}, 6.8 \mathrm{mmol})$ in acetonitrile $(50 \mathrm{~mL})$ was added, followed by $150 \mathrm{~mL}$ water. The solution was filtered and washed with water. Recrystallization from EtOH afforded $0.819 \mathrm{~g}(3.05 \mathrm{mmol}, 90 \%)$ of a yellow solid.

Data for 5. ${ }^{1} \mathrm{H} \mathrm{NMR}\left(300 \mathrm{MHz}, \mathrm{CDCl}_{3}\right) \delta 8.18(\mathrm{~d}, 2 \mathrm{H}, J=8.8 \mathrm{~Hz}$, aromatic), 7.37 (d, $2 \mathrm{H}, J=2.4 \mathrm{~Hz}$, aromatic), $6.94\left(\mathrm{dd}, 2 \mathrm{H}, J_{1}=8.8 \mathrm{~Hz}, J_{2}=2.4 \mathrm{~Hz}\right.$, aromatic), $3.95(\mathrm{~s}, 6 \mathrm{H}$, $\left.\mathrm{OCH}_{3}\right) ;{ }^{13} \mathrm{C} \mathrm{NMR}\left(75.5 \mathrm{MHz}, \mathrm{CDCl}_{3}\right) \delta 179.3,166.0,137.9,133.6,125.5,114.6,110.1$, 56.1; ESI-MS: $m / z=291\left([\mathrm{M}+\mathrm{Na}]^{+}\right)$; IR $(\mathrm{KBr}): v=3083,2976,2938,2843,1660,1594$, $1559,1499,1342,1310,1248,1230,1014,864 \mathrm{~cm}^{-1}$; UV-Vis $\left(\mathrm{CH}_{2} \mathrm{Cl}_{2}\right) \lambda_{\max }(\varepsilon)=238$ $\left(2.3 \times 10^{4}\right), 284\left(3.0 \times 10^{4}\right), 347\left(1.4 \times 10^{4}\right) \mathrm{nm}\left(\mathrm{L} \mathrm{mol}^{-1} \mathrm{~cm}^{-1}\right) ; \mathrm{Mp} .=226-230{ }^{\circ} \mathrm{C}$; Anal. Calc'd for $\mathrm{C}_{16} \mathrm{H}_{12} \mathrm{O}_{4}$ : C, 71.64; H, 4.51. Found: C, 71.90, H, 4.77.

Synthesis of 2,7-diiodo-3,6-dimethoxy-9,10-phenanthrenequinone (6). In a flask, $\mathbf{5}$ (3.99 g, $14.8 \mathrm{mmol}), \mathrm{I}_{2}(4.16 \mathrm{~g}, 16.3 \mathrm{mmol}), \mathrm{KIO}_{3}(1.27 \mathrm{~g}, 5.92 \mathrm{mmol}), \mathrm{H}_{2} \mathrm{O}(15.6 \mathrm{~mL})$, $\mathrm{H}_{2} \mathrm{SO}_{4}(1.56 \mathrm{~mL})$, and HOAc $(156 \mathrm{~mL})$ were combined and heated to reflux. After $5 \mathrm{~h}$, the solution was cooled, filtered and washed with copious amounts of $\mathrm{H}_{2} \mathrm{O}$. The solid was stirred in an aqueous $\mathrm{NaSO}_{3}$ solution, filtered and washed with EtOH. Recrystallization from ethanol gave a brown solid, and from acetone gave an orange solid. Yield: $6.68 \mathrm{~g}, 12.8 \mathrm{mmol}, 86 \%$

Data for 6. ${ }^{1} \mathrm{H}$ NMR $\left(300 \mathrm{MHz}, \mathrm{CDCl}_{3}\right) \delta 8.58(\mathrm{~s}, 2 \mathrm{H}$, aromatic $\mathrm{CH}), 7.17(\mathrm{~s}, 2 \mathrm{H}$, aromatic $\mathrm{CH}$ ), $4.10\left(\mathrm{~s}, 6 \mathrm{H}, \mathrm{OCH}_{3}\right) ;{ }^{13} \mathrm{C} \mathrm{NMR}\left(75.5 \mathrm{MHz}, \mathrm{CDCl}_{3}\right) \delta 177.5,164.1,142.5$, 137.5, 126.4, 104.5, 88.7, 57.1; ESI-MS: $m / z=543\left([\mathrm{M}+\mathrm{Na}]^{+}\right)$; IR $(\mathrm{KBr}): v=3027,2926$, $1670,1573,1492,1453,1330,1310,1268,1206,1058,1041,846,756 \mathrm{~cm}^{-1}$; UV-Vis $\left(\mathrm{CH}_{2} \mathrm{Cl}_{2}\right) \lambda_{\max }(\varepsilon)=241\left(1.5 \times 10^{4}\right), 306\left(6.3 \times 10^{4}\right), 345\left(1.3 \times 10^{4}\right) \mathrm{nm}\left(\mathrm{L} \mathrm{mol}^{-1} \mathrm{~cm}^{-1}\right)$; Mp. $>300{ }^{\circ} \mathrm{C}$; Anal. Calc'd for $\mathrm{C}_{16} \mathrm{H}_{10} \mathrm{O}_{4} \mathrm{I}_{2}$ : C, 36.95, H, 1.94. Found C, 37.35, H 2.14.

Synthesis of 9,10-dihexyloxy-2,7-diiodo-3,6-dimethoxyphenanthrene (7a). Compound 6 (1.015 g, $2.125 \mathrm{mmol}$ ) was dissolved in $50 \mathrm{~mL} \mathrm{DMF}$ and degassed with $\mathrm{N}_{2}$ for $5 \mathrm{~min}$. $\mathrm{Na}_{2} \mathrm{~S}_{2} \mathrm{O}_{4}$ (1.995 g, $\left.11.46 \mathrm{mmol}\right)$ was added to the solution and stirred for $15 \mathrm{~min}$, after which $\mathrm{K}_{2} \mathrm{CO}_{3}(1.175 \mathrm{~g}, 8.50 \mathrm{mmol}), \mathrm{Bu}_{4} \mathrm{NBr}(0.050 \mathrm{~g}, 0.154 \mathrm{mmol})$, and 1-bromohexane $(0.90 \mathrm{~mL}, 4.64 \mathrm{mmol})$ were added. The solution was heated for $16 \mathrm{~h}$ at $80{ }^{\circ} \mathrm{C}$. After 
cooling, the yellow solution was poured into $\mathrm{H}_{2} \mathrm{O}$ and extracted with EtOAc $(3 \times 125$ $\mathrm{mL}$ ). The organic layer was dried with $\mathrm{MgSO}_{4}$, filtered and dried under vacuum. Chromatography on silica with 1:3 DCM/hexanes afforded a colourless oil which was recrystallized from EtOH and DCM to give white crystals. Yield: $0.398 \mathrm{~g}, 0.576 \mathrm{mmol}$, $27 \%$

Data for 7a. ${ }^{1} \mathrm{H}$ NMR $\left(300 \mathrm{MHz}, \mathrm{CDCl}_{3}\right) \delta 8.62(\mathrm{~s}, 2 \mathrm{H}$, aromatic $\mathrm{CH}), 7.69(\mathrm{~s}, 2 \mathrm{H}$, aromatic $\mathrm{CH}), 4.12\left(\mathrm{t}, 4 \mathrm{H}, \mathrm{OCH}_{2}\right), 4.06\left(\mathrm{~s}, 6 \mathrm{H}, \mathrm{OCH}_{3}\right), 1.85(\mathrm{~m}, 4 \mathrm{H}$, hexyloxy chain), $1.55(\mathrm{~m}, 4 \mathrm{H}$, hexyloxy chain), 1.37 (m, 8H, hexyloxy chain), 0.89 (t, $6 \mathrm{H}$, hexyloxy chain); ${ }^{13} \mathrm{C}$ NMR $\left(75.5 \mathrm{MHz}, \mathrm{CDCl}_{3}\right) \delta 155.8,141.0,134.3,128.8,126.4,102.4,88.6,73.9,56.8$, 31.9, 30.6, 26.1, 22.9, 14.3; $\mathrm{R}_{\mathrm{F}}\left(1: 3 \mathrm{DCM} /\right.$ hexanes): 0.11 ; ESI-MS: $\mathrm{m} / \mathrm{z}=713\left([\mathrm{M}+\mathrm{Na}]^{+}\right)$; IR (KBr): $v=2999,2952,2925,2856,1589,1480,1453,1438,1409,1355,1240,1152$, $1080,1045,891,833,658 \mathrm{~cm}^{-1}$; UV-Vis $\left(\mathrm{CH}_{2} \mathrm{Cl}_{2}\right) \lambda_{\max }(\varepsilon)=232\left(3.7 \times 10^{4}\right), 269(5.9 \mathrm{x}$ $\left.10^{4}\right), 299\left(5.3 \times 10^{4}\right), 313\left(4.3 \times 10^{4}\right) \mathrm{nm}\left(\mathrm{L} \mathrm{mol}^{-1} \mathrm{~cm}^{-1}\right) ; \mathrm{Mp} .=78-81{ }^{\circ} \mathrm{C}$; Anal. Calc'd for $\mathrm{C}_{28} \mathrm{H}_{36} \mathrm{O}_{4} \mathrm{I}_{2}: \mathrm{C}, 48.71, \mathrm{H}$, 5.26. Found $\mathrm{C}, 48.91, \mathrm{H}, 5.43$.

Synthesis of 9,10-di-2-ethylhexyloxy-2,7-diiodo-3,6-dimethoxyphenanthrene (7b). Compound 6 (1.199 g, $2.31 \mathrm{mmol}$ ) was dissolved in $50 \mathrm{~mL}$ DMF and degassed with $\mathrm{N}_{2}$ for $5 \mathrm{~min}$. $\quad \mathrm{Na}_{2} \mathrm{~S}_{2} \mathrm{O}_{4}(1.204 \mathrm{~g}, 6.92 \mathrm{mmol})$ was added to the solution and stirred for 15 min, after which $\mathrm{K}_{2} \mathrm{CO}_{3}(1.274 \mathrm{~g}, 9.21 \mathrm{mmol}), \mathrm{Bu}_{4} \mathrm{NBr}(0.050 \mathrm{~g}, 0.154 \mathrm{mmol})$, and 2ethylhexylbromide $(1.23 \mathrm{~mL}, 6.92 \mathrm{mmol})$ were added. The solution was heated for $16 \mathrm{~h}$ at $80{ }^{\circ} \mathrm{C}$. After cooling, the yellow solution was poured into $\mathrm{H}_{2} \mathrm{O}$ and extracted with EtOAc $(3 \times 125 \mathrm{~mL})$. The organic layer was dried with $\mathrm{MgSO}_{4}$, filtered and dried under vacuum. Chromatography on silica with 1:3 DCM/hexanes afforded a colourless oil which was recrystallized from EtOH and DCM to give white crystals. Yield: $0.750 \mathrm{~g}$, $1.00 \mathrm{mmol}, 44 \%$

Data for 7b. ${ }^{1} \mathrm{H}$ NMR $\left(300 \mathrm{MHz}, \mathrm{CDCl}_{3}\right) \delta 8.64(\mathrm{~s}, 2 \mathrm{H}$, aromatic $\mathrm{CH}), 7.71(\mathrm{~s}, 2 \mathrm{H}$, aromatic $\mathrm{CH}), 4.07\left(\mathrm{~s}, 6 \mathrm{H}, \mathrm{OCH}_{3}\right) 4.00\left(\mathrm{~d}, 4 \mathrm{H}, \mathrm{OCH}_{2}\right), 1.83(\mathrm{~m}, 2 \mathrm{H}, \mathrm{CH}), 1.57(\mathrm{~m}, 4 \mathrm{H}$, ethex chain), $1.36(\mathrm{~m}, 12 \mathrm{H}$, ethex chain $), 0.96\left(\mathrm{~m}, 12 \mathrm{H}\right.$, ethex $\left.\mathrm{CH}_{3}\right) ;{ }^{13} \mathrm{C} \mathrm{NMR}(100.6$ $\left.\mathrm{MHz}_{2} \mathrm{CDCl}_{3}\right) \delta 155.8,141.3,134.3,128.9,126.3,102.5,88.4,76.7,56.8,40.8,30.8$, 29.4, 24.0, 23.4, 14.4, 11.4; $\mathrm{R}_{\mathrm{F}}\left(1: 3 \mathrm{DCM} /\right.$ hexanes): 0.14; ESI-MS: $m / z=769\left([\mathrm{M}+\mathrm{Na}]^{+}\right)$; IR (KBr): $v=3001,2959,2926,2871,1589,1464,1439,1355,1241,1144,1043,886$, $832,656 \mathrm{~cm}^{-1}$; UV-Vis $\left(\mathrm{CH}_{2} \mathrm{Cl}_{2}\right) \lambda_{\max }(\varepsilon)=232\left(2.9 \times 10^{4}\right), 269\left(4.8 \times 10^{4}\right), 299(4.5 \mathrm{x}$ $\left.10^{4}\right), 313\left(3.7 \times 10^{4}\right) \mathrm{nm}\left(\mathrm{L} \mathrm{mol}^{-1} \mathrm{~cm}^{-1}\right)$; Mp. $=110-112{ }^{\circ} \mathrm{C}$; Anal. Calc'd for $\mathrm{C}_{32} \mathrm{H}_{44} \mathrm{O}_{4} \mathrm{I}_{2}$ : C, 51.49, H, 5.94. Found C, 51.69, H 6.18.

Synthesis of 9,10-dibenzyloxy-2,7-diiodo-3,6-dimethoxyphenanthrene (7c). Compound 6 (0.157 g, $0.302 \mathrm{mmol})$ was dissolved in $20 \mathrm{~mL}$ DMF and degassed with $\mathrm{N}_{2}$ for $5 \mathrm{~min}$. $\quad \mathrm{Na}_{2} \mathrm{~S}_{2} \mathrm{O}_{4}(0.158 \mathrm{~g}, 0.907 \mathrm{mmol})$ was added to the solution and stirred for 15 min, after which $\mathrm{K}_{2} \mathrm{CO}_{3}(0.405 \mathrm{~g}, 2.93 \mathrm{mmol}), \mathrm{Bu}_{4} \mathrm{NBr}(0.050 \mathrm{~g}, 0.154 \mathrm{mmol})$, and benzyl chloride $(0.5 \mathrm{~mL}, 4.34 \mathrm{mmol})$ were added. The solution was heated for $16 \mathrm{~h}$ at $80{ }^{\circ} \mathrm{C}$. After cooling, the yellow solution was poured into $\mathrm{H}_{2} \mathrm{O}$ and extracted with EtOAc $(3 \times 75$ $\mathrm{mL}$ ). The organic layer was dried with $\mathrm{MgSO}_{4}$, filtered and dried under vacuum. 
Chromatography on silica with 1:3 DCM/hexanes afforded white crystals. Yield: $0.073 \mathrm{~g}$, $0.104 \mathrm{mmol}, 34 \%$

Data for 7c. ${ }^{1} \mathrm{H}$ NMR $\left(400 \mathrm{MHz}, \mathrm{CDCl}_{3}\right) \delta 8.62(\mathrm{~s}, 2 \mathrm{H}$, aromatic $\mathrm{CH}), 7.70(\mathrm{~s}, 2 \mathrm{H}$, aromatic $\mathrm{CH}), 7.47(\mathrm{~d}, 4 \mathrm{H}$, aromatic $\mathrm{CH}), 7.36(\mathrm{~m}, 6 \mathrm{H}$, aromatic $\mathrm{CH}), 5.21(\mathrm{~s}, 4 \mathrm{H}$, $\left.\mathrm{OCH}_{2}\right), 4.07\left(\mathrm{~s}, 6 \mathrm{H}, \mathrm{OCH}_{3}\right) ;{ }^{13} \mathrm{C} \mathrm{NMR}\left(100.6 \mathrm{MHz}, \mathrm{CDCl}_{3}\right) \delta 156.1,141.2,, 137.3$, 134.5, 129.1, 128.8, 128.7, 128.5, 126.1, 102.4, 88.7, 75.9, 56.8; EI-MS: $m / z=702\left(\mathrm{M}^{+}\right)$; IR $(\mathrm{KBr}): v=2999,2932,2875,2855,1586,1455,1436,1350,1275,1132,1044,955$, $896,841,758,696 \mathrm{~cm}^{-1}$; UV-Vis $\left(\mathrm{CH}_{2} \mathrm{Cl}_{2}\right) \lambda_{\max }(\varepsilon)=233\left(3.2 \times 10^{4}\right), 270\left(5.0 \times 10^{4}\right), 299$ $\left(4.7 \times 10^{4}\right), 312\left(3.7 \times 10^{4}\right) \mathrm{nm}\left(\mathrm{L} \mathrm{mol}^{-1} \mathrm{~cm}^{-1}\right)$; Mp. $213-215^{\circ} \mathrm{C}$.

Synthesis of Model Compound 8. Compound 7a (0.153 g, 0.222 mmol), phenylacetylene $(0.2 \mathrm{~mL}, 1.82 \mathrm{mmol})$ and $\mathrm{CuI}(30 \mathrm{mg}, 0.16 \mathrm{mmol})$ were dissolved in 10 $\mathrm{mL}$ toluene and $4 \mathrm{~mL}$ diisopropylamine. After the solution was degassed, $\mathrm{Pd}\left(\mathrm{PPh}_{3}\right)_{4}(50$ $\mathrm{mg}, 0.043 \mathrm{mmol}$ ) was added. The reaction was heated for $1 \mathrm{~h}$ at $50{ }^{\circ} \mathrm{C}$. Upon cooling, the solution was poured into water and extracted with DCM. The organic layer was dried with $\mathrm{MgSO}_{4}$, filtered and dried under vacuum to obtain a brown oil. The product was chromatographed with 3:1 hexanes/DCM to obtain a yellow oil. Recrystallization from EtOH and DCM gave yellow needles. Yield: $0.114 \mathrm{~g}, 0.178 \mathrm{mmol}, 80 \%$

Data for 8. ${ }^{1} \mathrm{H}$ NMR $\left(400 \mathrm{MHz}, \mathrm{CDCl}_{3}\right) \delta 8.33(\mathrm{~s}, 2 \mathrm{H}$, aromatic $\mathrm{CH}), 7.79(\mathrm{~s}, 2 \mathrm{H}$, aromatic $\mathrm{CH}), 7.61(\mathrm{~m}, 4 \mathrm{H}$, aromatic $\mathrm{CH}), 7.35(\mathrm{~m}, 6 \mathrm{H}$, aromatic $\mathrm{CH}), 4.17(\mathrm{t}, 4 \mathrm{H}$, $\left.\mathrm{OCH}_{2}\right), 4.12\left(\mathrm{~s}, 6 \mathrm{H}, \mathrm{OCH}_{3}\right), 1.89(\mathrm{~m}, 4 \mathrm{H}$, hexyloxy chain), $1.51(\mathrm{~m}, 4 \mathrm{H}$, hexyloxy chain), $1.38\left(\mathrm{~m}, 8 \mathrm{H}\right.$, hexyloxy chain), $0.91\left(\mathrm{t}, 6 \mathrm{H}\right.$, hexyloxy chain); ${ }^{13} \mathrm{C}$ NMR $(75.5 \mathrm{MHz}$, $\left.\mathrm{CDCl}_{3}\right) \delta 157.6,141.7,132.0,128.7,128.5,124.9,123.6,114.3,102.9,94.4,86.3,73.9$, 56.4, 31.9, 30.6, 26.2, 23.0, 14.3; ESI-MS: $m / z=662\left([\mathrm{M}+\mathrm{Na}]^{+}\right)$; IR $(\mathrm{KBr}): v=2953$, 2930, 2854, 1591, 1499, 1443, 1365, 1235, 1118, 1009, 832, 756, $694 \mathrm{~cm}^{-1}$; UV-Vis $\left(\mathrm{CH}_{2} \mathrm{Cl}_{2}\right) \lambda_{\max }(\varepsilon)=295\left(4.0 \times 10^{4}\right), 353\left(4.4 \times 10^{4}\right) \mathrm{nm}\left(\mathrm{L} \mathrm{mol}^{-1} \mathrm{~cm}^{-1}\right) ; \mathrm{Mp} .=70-72{ }^{\circ} \mathrm{C}$; Anal. Calc'd for $\mathrm{C}_{44} \mathrm{H}_{46} \mathrm{O}_{4}$ : C, 82.72 , H, 7.26. Found C, 82.40, H, 7.29. Fluorescence $\left(\mathrm{CH}_{2} \mathrm{Cl}_{2}\right): \lambda_{\mathrm{em}}=456 \mathrm{~nm}\left(\lambda_{\mathrm{exc}}=354 \mathrm{~nm}\right)$.

Synthesis of Polymer 10. Compound $7 \mathbf{b}(0.2035 \mathrm{~g}, 0.2726 \mathrm{mmol})$ and 1,4-diethynyl2,5-dihexadecyloxybenzene $9(0.1688 \mathrm{~g}, 0.2781 \mathrm{mmol})$ were dissolved in $28 \mathrm{~mL}$ of a 5:2 $\mathrm{THF} /$ diisopropylamine solution and degassed by three freeze/pump/thaw cycles. CuI (30 $\mathrm{mg}, 0.16 \mathrm{mmol})$ and $\mathrm{Pd}\left(\mathrm{PPh}_{3}\right)_{4}(50 \mathrm{mg}, 0.043 \mathrm{mmol})$ were added to the solution in a nitrogen filled glovebox and the mixture underwent 2 additional freeze/pump/thaw cycles. The solution was heated to reflux under vacuum for $16 \mathrm{~h}$ to obtain a brown solution. The solution was filtered and the solvent was removed by vacuum. After redissolution in $\mathrm{DCM}$, the solution was washed with aqueous $\mathrm{KCN}$, then water. The solvent was evaporated until the polymer solution was viscous, and the solution was then precipitated in $\mathrm{MeOH}$ and filtered to obtain a yellow solid. This was repeated twice to remove impurities. Yield: $0.256 \mathrm{~g}, 86 \%$. The ${ }^{1} \mathrm{H}$ NMR spectrum was broad and only alkyl resonances could be observed in the ${ }^{13} \mathrm{C}$ spectrum. 
Data for 10. ${ }^{1} \mathrm{H}$ NMR $\left(300 \mathrm{MHz}, \mathrm{CDCl}_{3}\right) \delta 8.35$ (s, aromatic $\left.\mathrm{CH}\right), 7.10(\mathrm{~s}$, aromatic $\mathrm{CH})$, 4.14, 4.09 (m, alkoxy), 2.0-0.8 (br m, alkoxy); ${ }^{13} \mathrm{C} \mathrm{NMR} \mathrm{(75.5} \mathrm{MHz,} \mathrm{CDCl}_{3}$ ) $\delta 40.9,32.2$, $30.8,29.9,29.7,29.6,29.4,26.2,24.1,23.5,22.9,14.4,14.3,11.5$, resonances from the aromatic region of the spectrum were not observed; IR (KBr): $v=3028,2924,2851$, 1736, 1603, 1494, 1453, 1211, 1025, 756, 697, $536 \mathrm{~cm}^{-1}$; UV-Vis $\left(\mathrm{CH}_{2} \mathrm{Cl}_{2}\right) \lambda_{\max }=304$, $437 \mathrm{~nm}$; UV-Vis (film) $\lambda_{\max }=449 \mathrm{~nm}$; decomposition begins at $150{ }^{\circ} \mathrm{C}$; Anal. Calc'd for $\mathrm{C}_{74} \mathrm{H}_{112} \mathrm{O}_{6}: \mathrm{C}, 80.97, \mathrm{H}, 10.28$. Found: $\mathrm{C}, 80.89$, H, 10.00. GPC: $\mathrm{M}_{\mathrm{n}}=146$ 142, $\mathrm{M}_{\mathrm{w}}=1$ 368420 , PDI $=9.36$. Fluorescence $\left(\mathrm{CH}_{2} \mathrm{Cl}_{2}\right): \lambda_{\text {em }}=455 \mathrm{~nm}\left(\lambda_{\text {exc }}=438 \mathrm{~nm}\right), \Phi=70 \%$. Fluorescence (film): $\lambda_{\mathrm{em}}=470 \mathrm{~nm}\left(\lambda_{\mathrm{exc}}=449 \mathrm{~nm}\right)$.

Synthesis of Model Compound 11. To a mixture of $7 \mathbf{b}(0.205 \mathrm{~g}, 0.274 \mathrm{mmol})$, $\mathrm{Pd}(\mathrm{OAc})_{2}(0.005 \mathrm{~g}, 0.022 \mathrm{mmol})$ and tri(o-tolyl)phosphine $(0.013 \mathrm{~g}, 0.043 \mathrm{mmol})$ were added anhydrous DMF $(5 \mathrm{~mL})$, triethylamine $(3 \mathrm{~mL})$ and styrene $(0.3 \mathrm{~mL}, 2.6 \mathrm{mmol})$. The solution was heated at $130{ }^{\circ} \mathrm{C}$ for $20 \mathrm{~h}$, after which it was diluted with $\mathrm{CHCl}_{3}$ and washed with water. After drying with $\mathrm{MgSO}_{4}$ and removing the solvent by evaporation, the residue was chromatographed using 3:1 hexanes/DCM to afford a yellow oil. Yield: $0.101 \mathrm{~g}, 0.145 \mathrm{mmol}, 53 \%$.

Data for 11. ${ }^{1} \mathrm{H}$ NMR $\left(400 \mathrm{MHz}, \mathrm{CDCl}_{3}\right) \delta 8.46(\mathrm{~s}, 2 \mathrm{H}$, aromatic $\mathrm{CH}), 7.79(\mathrm{~s}, 2 \mathrm{H}$, aromatic $\mathrm{CH}), 7.66-7.58(\mathrm{~m}, 6 \mathrm{H}$, aromatic $\mathrm{CH}), 7.40-7.20(\mathrm{~m}, 8 \mathrm{H}$, aromatic $\mathrm{CH}), 4.11(\mathrm{~d}$, $\left.4 \mathrm{H}, \mathrm{OCH}_{2}\right), 4.10\left(\mathrm{~s}, 6 \mathrm{H}, \mathrm{OCH}_{3}\right), 1.91(\mathrm{~m}, 2 \mathrm{H}, \mathrm{CH}), 1.81-1.34(\mathrm{~m}, 18 \mathrm{H}$, ethex chain), 1.05 (t, 6H, ethex chain), $0.91\left(\mathrm{~m}, 6 \mathrm{H}\right.$, ethex $\left.\mathrm{CH}_{3}\right) ;{ }^{13} \mathrm{C} \mathrm{NMR}\left(75.5 \mathrm{MHz}, \mathrm{CDCl}_{3}\right) \delta 155.7$, 142.2 , 138.2, 130.3, 128.9, 128.6, 127.8, 127.7, 126.9, 124.6, 123.7, 120.5, 102.8, 76.7, 56.0, 41.0, 31.0, 29.6, 24.2, 23.5, 14.4, 11.5; EI-MS: $\mathrm{m} / \mathrm{z}=698\left(\mathrm{M}^{+}\right)$; IR $(\mathrm{NaCl}): v=$ 2956, 2928, 2873, 2855, 1598, 1498, 1447, 1370, 1240, 1220, 1143, 1014, 965, 829, 752, $693 \mathrm{~cm}^{-1}$; UV-Vis $\left(\mathrm{CH}_{2} \mathrm{Cl}_{2}\right) \lambda_{\max }(\varepsilon) 235\left(3.1 \times 10^{4}\right), 301\left(4.6 \times 10^{4}\right), 362\left(4.6 \times 10^{4}\right) \mathrm{nm}$ $\left(\mathrm{L} \mathrm{mol}{ }^{-1} \mathrm{~cm}^{-1}\right)$; High Res MS Calc'd for $\mathrm{C}_{48} \mathrm{H}_{58} \mathrm{O}_{4}$ : 698.43351. Found: 698.43323 . Fluorescence $\left(\mathrm{CH}_{2} \mathrm{Cl}_{2}\right): \lambda_{\mathrm{em}}=475 \mathrm{~nm}\left(\lambda_{\mathrm{exc}}=368 \mathrm{~nm}\right)$.

Synthesis of Polymer 13. Monomers $7 \mathbf{b}(0.0642 \mathrm{~g}, 0.086 \mathrm{mmol})$ and $12(0.0526 \mathrm{~g}$, $0.086 \mathrm{mmol}$ ) were combined in a $100 \mathrm{~mL}$ Schlenk tube. To these solids were added $5 \mathrm{~mL}$ of dry $\mathrm{DMF}$ and $1 \mathrm{~mL}$ of distilled $\mathrm{Bu}_{3} \mathrm{~N}$ via syringe. The reaction mixture was then degassed by three freeze/pump/thaw cycles. In a nitrogen filled glovebox, $\mathrm{Pd}(\mathrm{OAc})_{2}(50$ $\mathrm{mg}, 0.043 \mathrm{mmol})$ and (o-tol $)_{3} \mathrm{P}(0.013 \mathrm{~g}, 0.043 \mathrm{mmol})$ were added. The reaction mixture was subsequently freeze/pump/ thawed one more time. A fluorescent green solution was obtained after heating the reaction mixture at $85{ }^{\circ} \mathrm{C}$ for $40 \mathrm{~h}$. After cooling to room temperature, the solution was filtered and precipitated into $200 \mathrm{~mL}$ of $\mathrm{MeOH}$ to yield a yellow precipitate. The polymer was further purified by precipitating the polymer from $\mathrm{DCM}$ into $\mathrm{MeOH}$. The above procedure gave $0.095 \mathrm{~g}$ of polymer 13 in $45 \%$ yield. The ${ }^{1} \mathrm{H}$ NMR spectrum was broad and only alkyl resonances could be observed in the ${ }^{13} \mathrm{C}$ spectrum.

Data for 13. ${ }^{1} \mathrm{H}$ NMR $\left(300 \mathrm{MHz}, \mathrm{CDCl}_{3}\right) \delta 8.46$ (br), 7.80 (br), 7.69 (br), 7.21 (br), 6.86.6 (br), 4.2-3.7 (br), 2.0-0.8 (br); ${ }^{13} \mathrm{C} \mathrm{NMR} \mathrm{(100.6} \mathrm{MHz,} \mathrm{CDCl}_{3}$ ) $\delta$ 56.0, 40.9, 32.1, 29.9, $29.5,26.4,23.4,22.9,14.3,11.4$, resonances from the aromatic region of the spectrum were not observed; IR $(\mathrm{NaCl}): v=2955,2923,2852,1728,1677,1598,1502,1467,1422$, 
1364, 1239, 1204, 1146, $1016 \mathrm{~cm}^{-1}$; UV-Vis $\left(\mathrm{CH}_{2} \mathrm{Cl}_{2}\right) \lambda_{\max }=431 \mathrm{~nm} ; \mathrm{GPC}: \mathrm{M}_{\mathrm{n}}=4439$, $\mathrm{M}_{\mathrm{w}}=8702, \mathrm{PDI}=1.96$. Fluorescence $\left(\mathrm{CH}_{2} \mathrm{Cl}_{2}\right): \lambda_{\mathrm{em}}=499 \mathrm{~nm}\left(\lambda_{\text {exc }}=441 \mathrm{~nm}\right), \Phi=59 \%$.

Synthesis of 9,10-diethylhexyloxy-3,6-dimethoxy-2,7-divinylphenanthrene (14). A solution of tributyl(vinyl)tin $(0.84 \mathrm{~mL}, 2.6 \mathrm{mmol})$ in DMF $(40 \mathrm{~mL})$ was added to a mixture of $7 \mathbf{b}(0.714 \mathrm{~g}, 0.957 \mathrm{mmol})$ and $\mathrm{Pd}\left(\mathrm{PPh}_{3}\right)_{2} \mathrm{Cl}_{2}(0.067 \mathrm{~g}, 0.095 \mathrm{mmol})$. The resulting solution was heated to $80{ }^{\circ} \mathrm{C}$ for $3 \mathrm{~h}$ and cooled. Ether $(100 \mathrm{~mL})$ was added to the reaction and the solution was washed $(3 \times 100 \mathrm{~mL})$ with aqueous $\mathrm{NH}_{4} \mathrm{Cl}$. The organic layer was flashed through silica and the solvent was removed under vacuum. Chromatography with 7:1 hexanes/DCM afforded a pale yellow oil. Yield: $0.237 \mathrm{~g}$, $0.433 \mathrm{mmol}, 45 \%$.

Data for 14. ${ }^{1} \mathrm{H}$ NMR $\left(300 \mathrm{MHz}, \mathrm{CDCl}_{3}\right) \delta 8.35(\mathrm{~s}, 2 \mathrm{H}$, aromatic $\mathrm{CH}), 7.76(\mathrm{~s}, 2 \mathrm{H}$, aromatic $\mathrm{CH}), 7.22\left(\mathrm{dd}, 2 \mathrm{H}, J_{\text {trans }}=17.7 \mathrm{~Hz}, J_{\text {cis }}=11.1 \mathrm{~Hz}, \mathrm{H}_{2} \mathrm{C}=\mathrm{CH}\right), 5.96\left(\mathrm{dd}, 2 \mathrm{H}, J_{\text {trans }}\right.$ $\left.=17.7 \mathrm{~Hz}, J_{\text {gem }}=1.5 \mathrm{~Hz}, \mathrm{C}=\mathrm{CH}_{\text {trans }}\right), 5.40\left(\mathrm{dd}, 2 \mathrm{H}, J_{\text {cis }}=11.1 \mathrm{~Hz}, J_{\text {gem }}=1.5 \mathrm{~Hz}, \mathrm{C}=\mathrm{CH}_{\text {cis }}\right)$, $4.08\left(\mathrm{~d}, 4 \mathrm{H}, \mathrm{OCH}_{2}\right), 4.05\left(\mathrm{~s}, 6 \mathrm{H}, \mathrm{OCH}_{3}\right), 1.89(\mathrm{~m}, 2 \mathrm{H}$, ethex chain), $1.63(\mathrm{~m}, 8 \mathrm{H}$, ethex chain), 1.40 ( $\mathrm{m}, 8 \mathrm{H}$, ethex chain), $1.02\left(\mathrm{t}, 6 \mathrm{H}\right.$, ethex chain), $0.94\left(\mathrm{t}, 6 \mathrm{H}\right.$, ethex chain); ${ }^{13} \mathrm{C}$ NMR $\left(75.5 \mathrm{MHz}, \mathrm{CDCl}_{3}\right) \delta 155.5,142.2,132.1,130.4,128.7,128.8,124.5,120.8,115.6$, $102.6,76.6,55.9,41.0,30.9,29.5,24.1,23.4,14.3,11.4$; ESI-MS: $m / z=569\left([\mathrm{M}+\mathrm{Na}]^{+}\right)$; IR $(\mathrm{NaCl}) v=2959,2923,2873,2855,1601,1484,1456,1446,1368,1236,1142,1047$, 997, 901, $834 \mathrm{~cm}^{-1}$; UV-Vis $\left(\mathrm{CH}_{2} \mathrm{Cl}_{2}\right) \lambda_{\max }(\varepsilon)=284\left(6.2 \times 10^{4}\right), 318\left(3.3 \times 10^{4}\right) \mathrm{nm}(\mathrm{L}$ $\left.\mathrm{mol}^{-1} \mathrm{~cm}^{-1}\right)$; High Res MS Calc'd for $\mathrm{C}_{36} \mathrm{H}_{50} \mathrm{O}_{4}$ : 546.37091. Found: 546.37118. Fluorescence $\left(\mathrm{CH}_{2} \mathrm{Cl}_{2}\right): \lambda_{\mathrm{em}}=449 \mathrm{~nm}\left(\lambda_{\mathrm{exc}}=318 \mathrm{~nm}\right)$.

Attempted polymerization of 14. To a mixture of $7 \mathbf{b}(0.073 \mathrm{~g}, 0.098 \mathrm{mmol}), 14(0.059$ g, $0.11 \mathrm{mmol}), \mathrm{Pd}(\mathrm{OAc})_{2}(0.001 \mathrm{~g}, 0.0045 \mathrm{mmol})$ and $\mathrm{PPh}_{3}(0.010 \mathrm{~g}, 0.038 \mathrm{mmol})$ were added anhydrous DMF $(4 \mathrm{~mL})$ and triethylamine $(2 \mathrm{~mL})$. The resulting yellow solution was heated to reflux for $48 \mathrm{~h}$, after which it was filtered and precipitated into $\mathrm{MeOH}$ to obtain a yellow solid. A second attempt at precipitation was unsuccessful and the product was recovered through evaporation of the solvent.

Data for 15. ${ }^{1} \mathrm{H}$ NMR (300 MHz, $\left.\mathrm{CDCl}_{3}\right) \delta 7.7$ (br), 7.5 (br), 7.2 (br), 4.2 (br), 4.1 (br), 2.3-0.5 (br). IR (NaCl) $v=2954,2929,2873,2860,1725,1594,1461,1368,1265,1239$, $1131,1017 \mathrm{~cm}^{-1}$; GPC: $\mathrm{M}_{\mathrm{n}}=2238, \mathrm{M}_{\mathrm{w}}=3313$. 

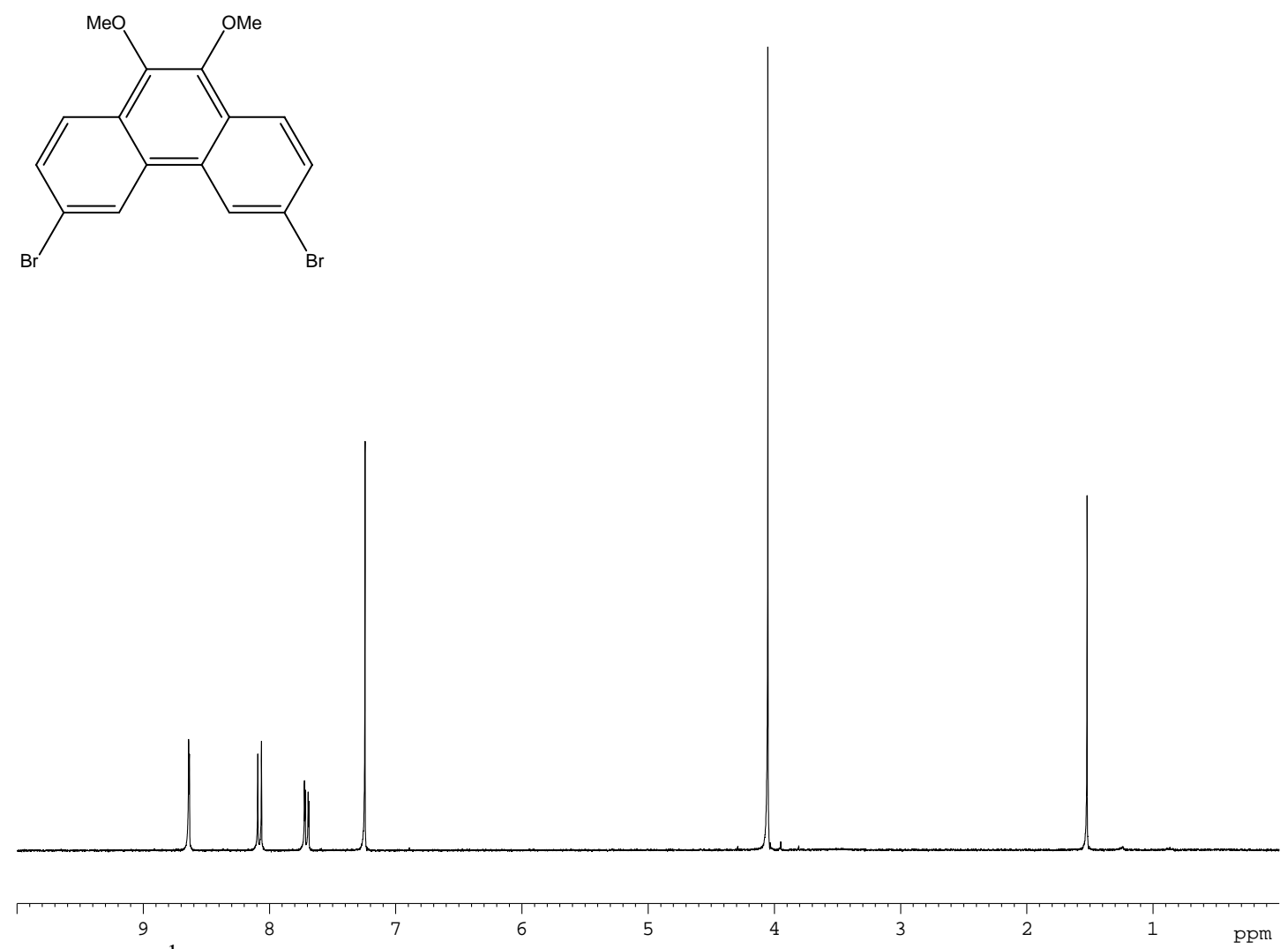

Figure 1. ${ }^{1} \mathrm{H}$ NMR spectrum of 3 .

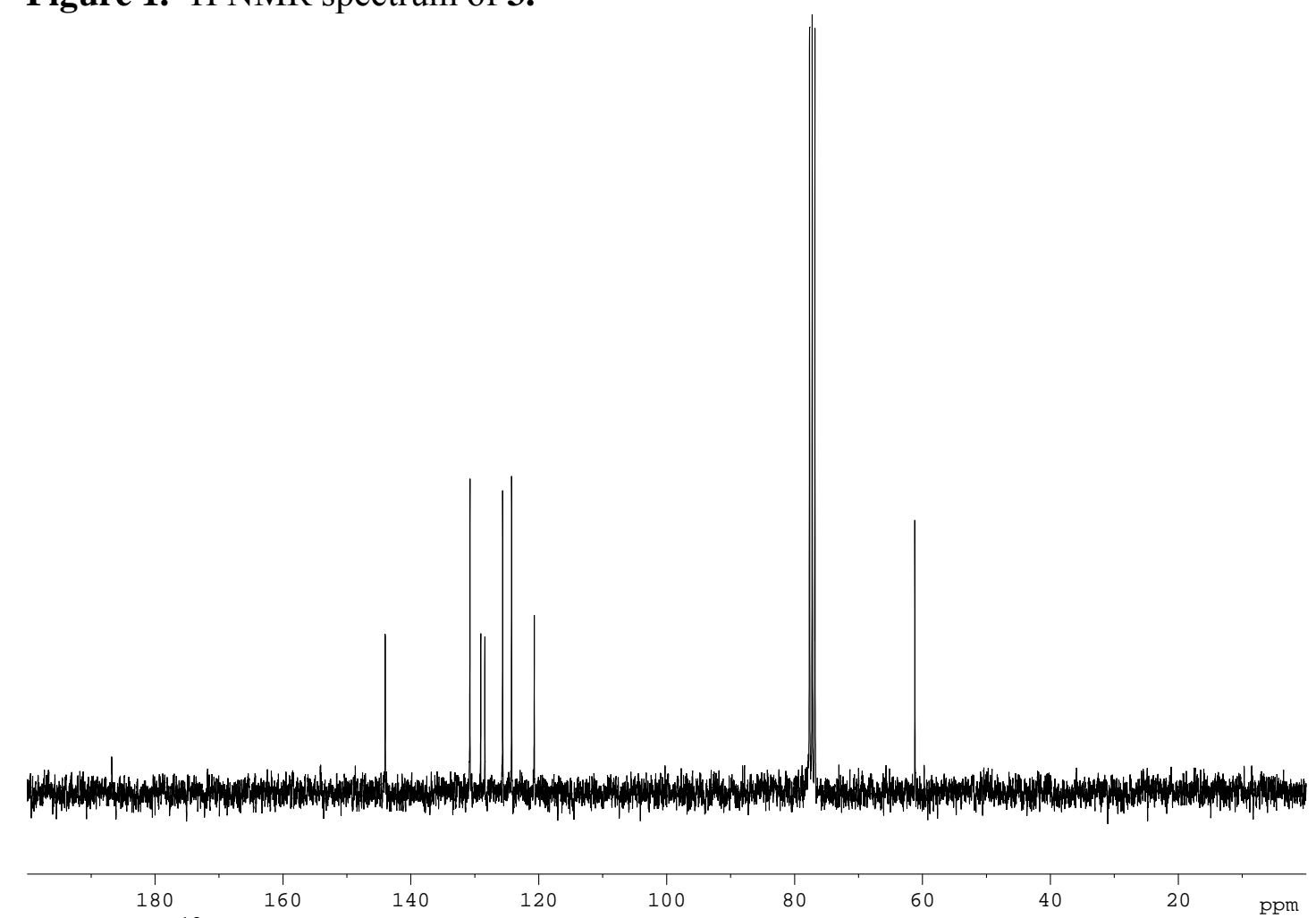

Figure 2. ${ }^{13} \mathrm{C}$ NMR spectrum of 3 . 

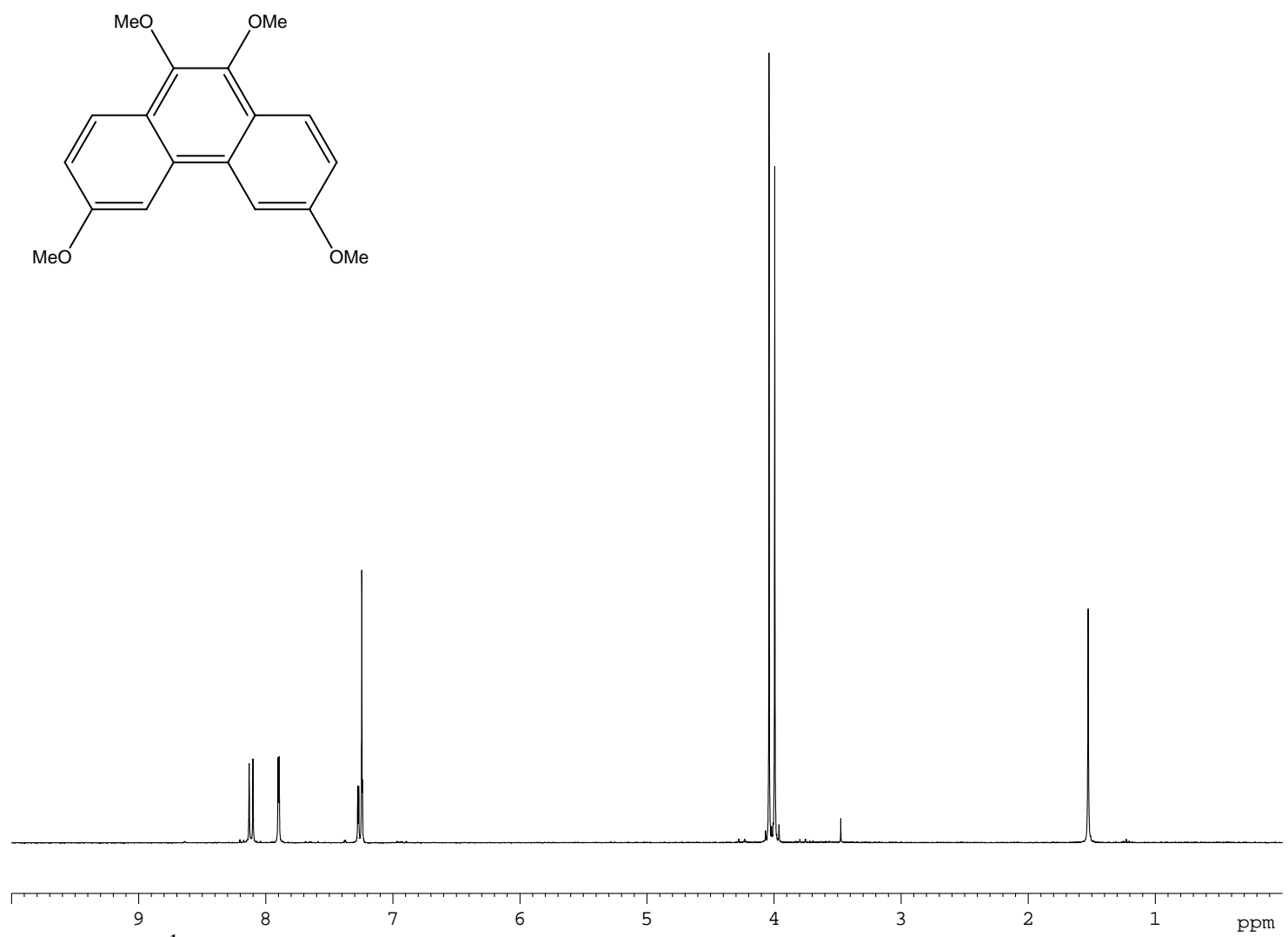

Figure 3. ${ }^{1} \mathrm{H}$ NMR spectrum of 4 .

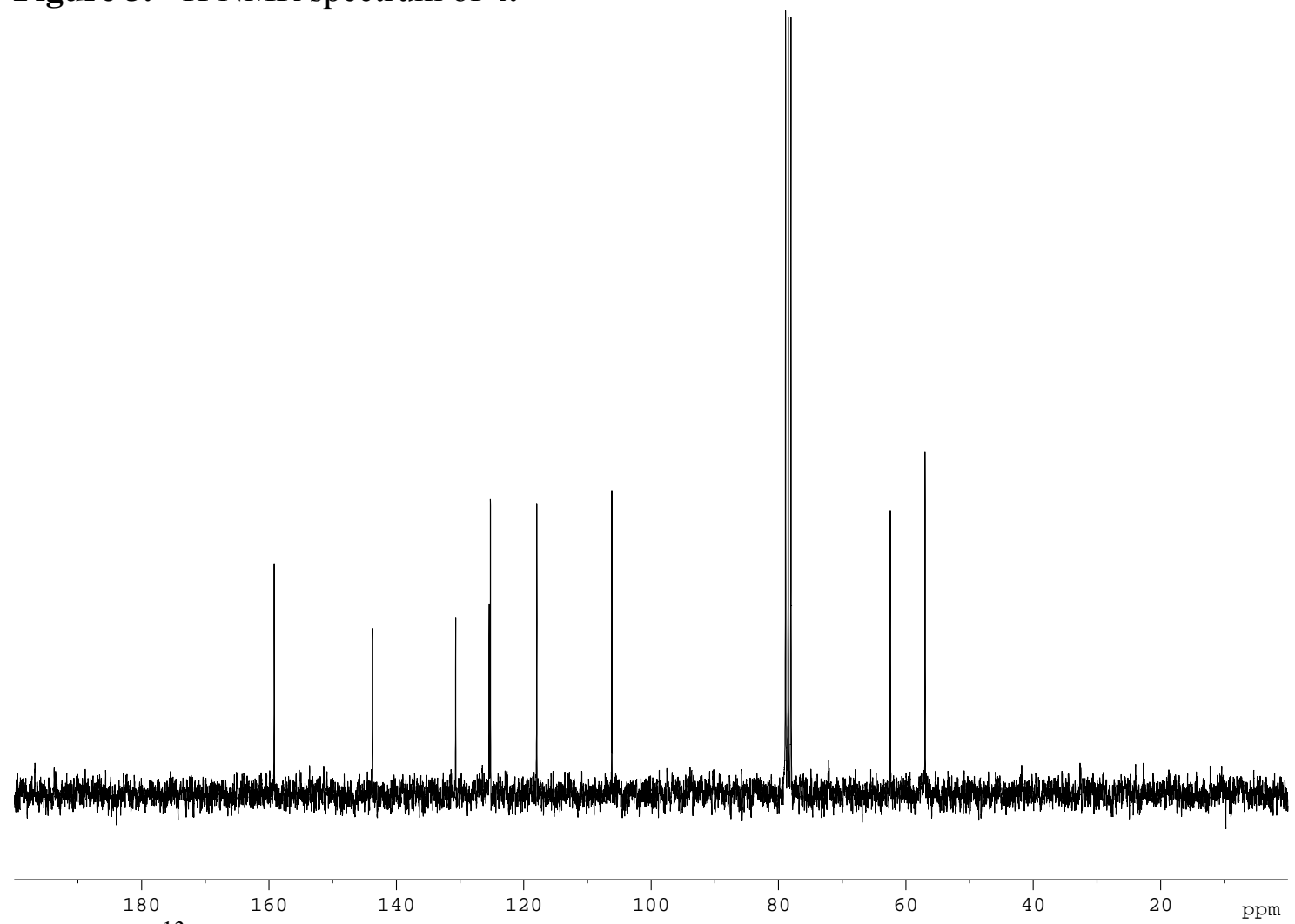

Figure $4 .{ }^{13} \mathrm{C}$ NMR spectrum of 4 . 


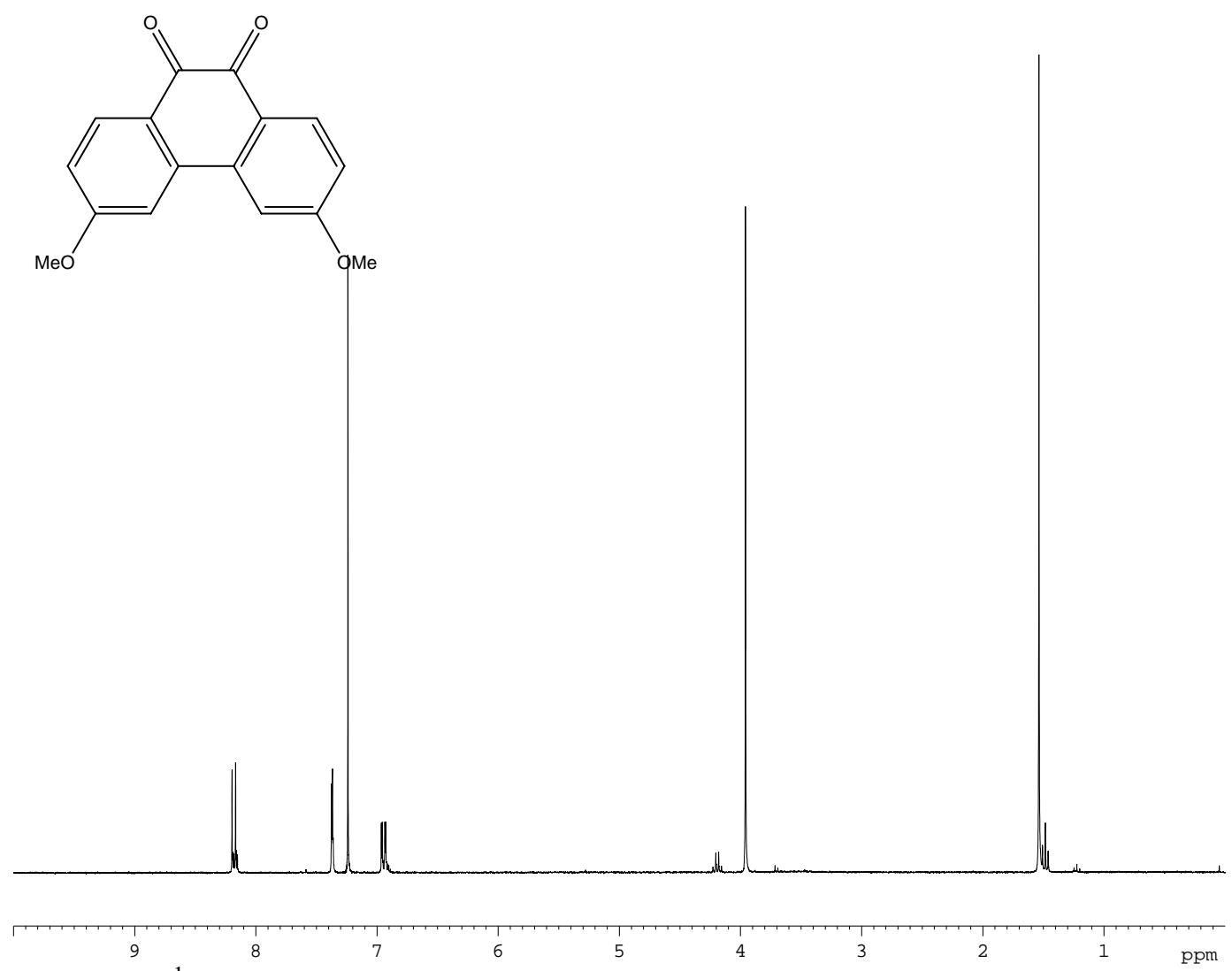

Figure 5. ${ }^{1} \mathrm{H}$ NMR spectrum of 5 .

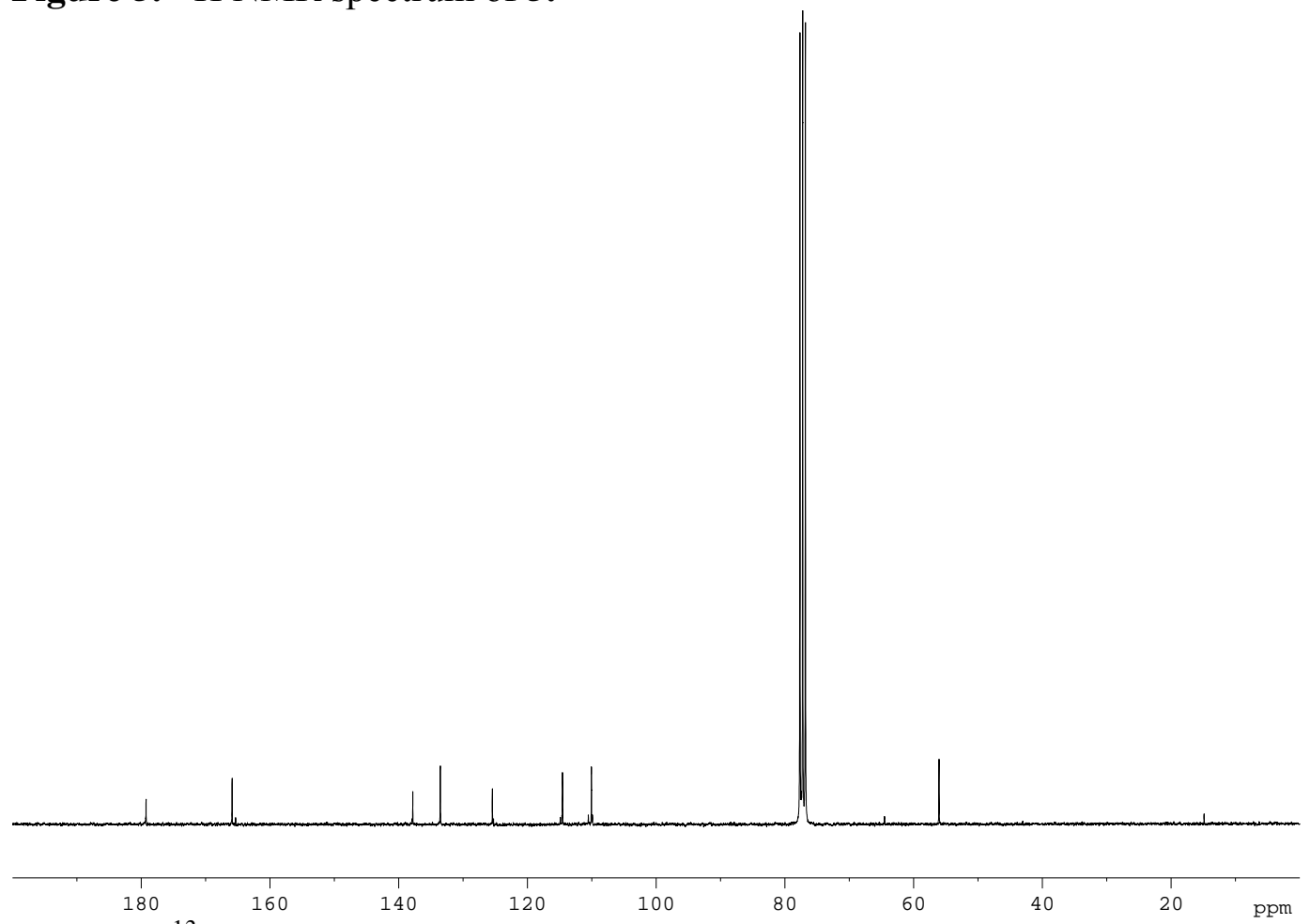

Figure 6. ${ }^{13} \mathrm{C}$ NMR spectrum of 5 . 


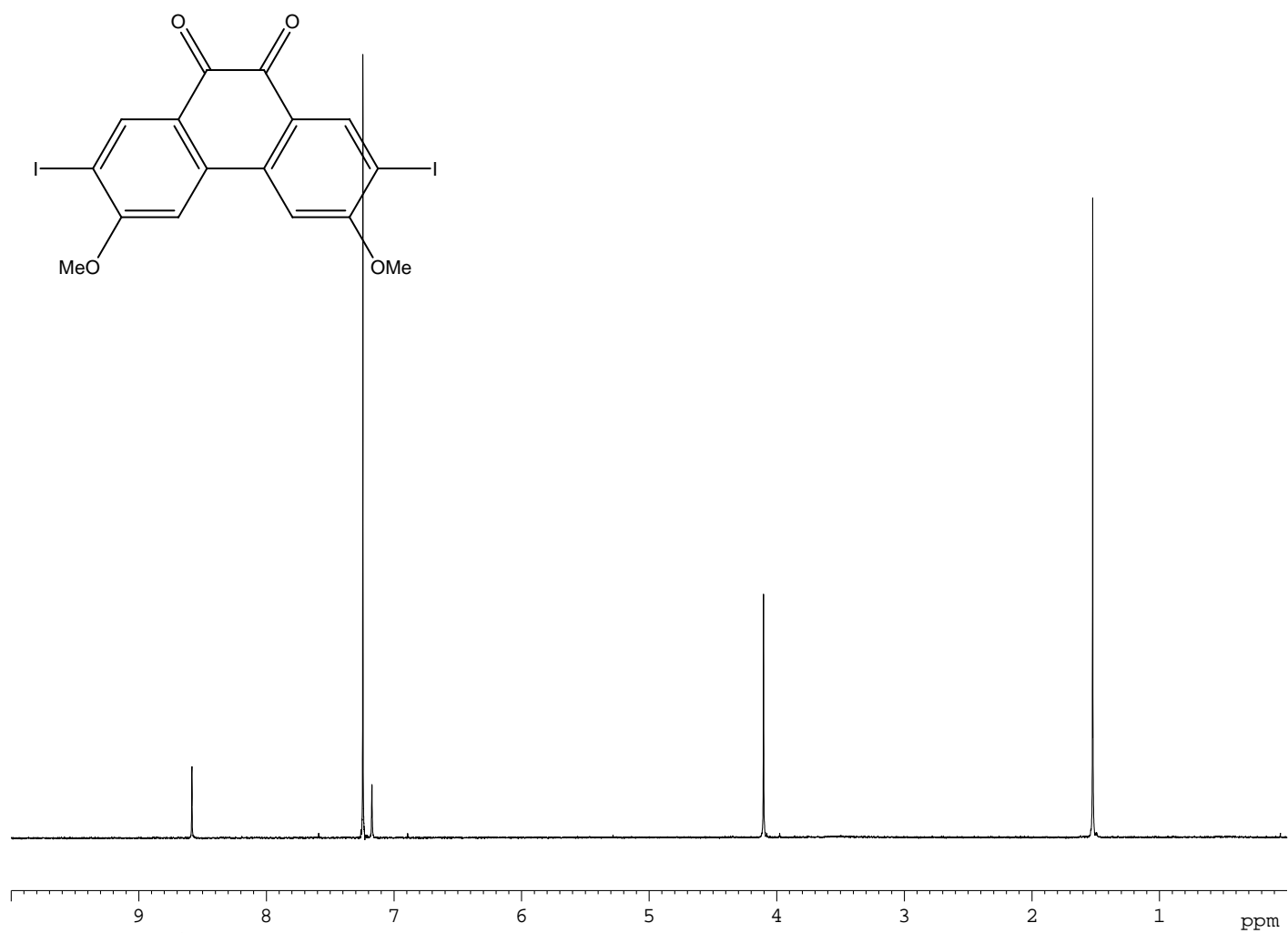

Figure 7. ${ }^{1} \mathrm{H}$ NMR spectrum of 6 .

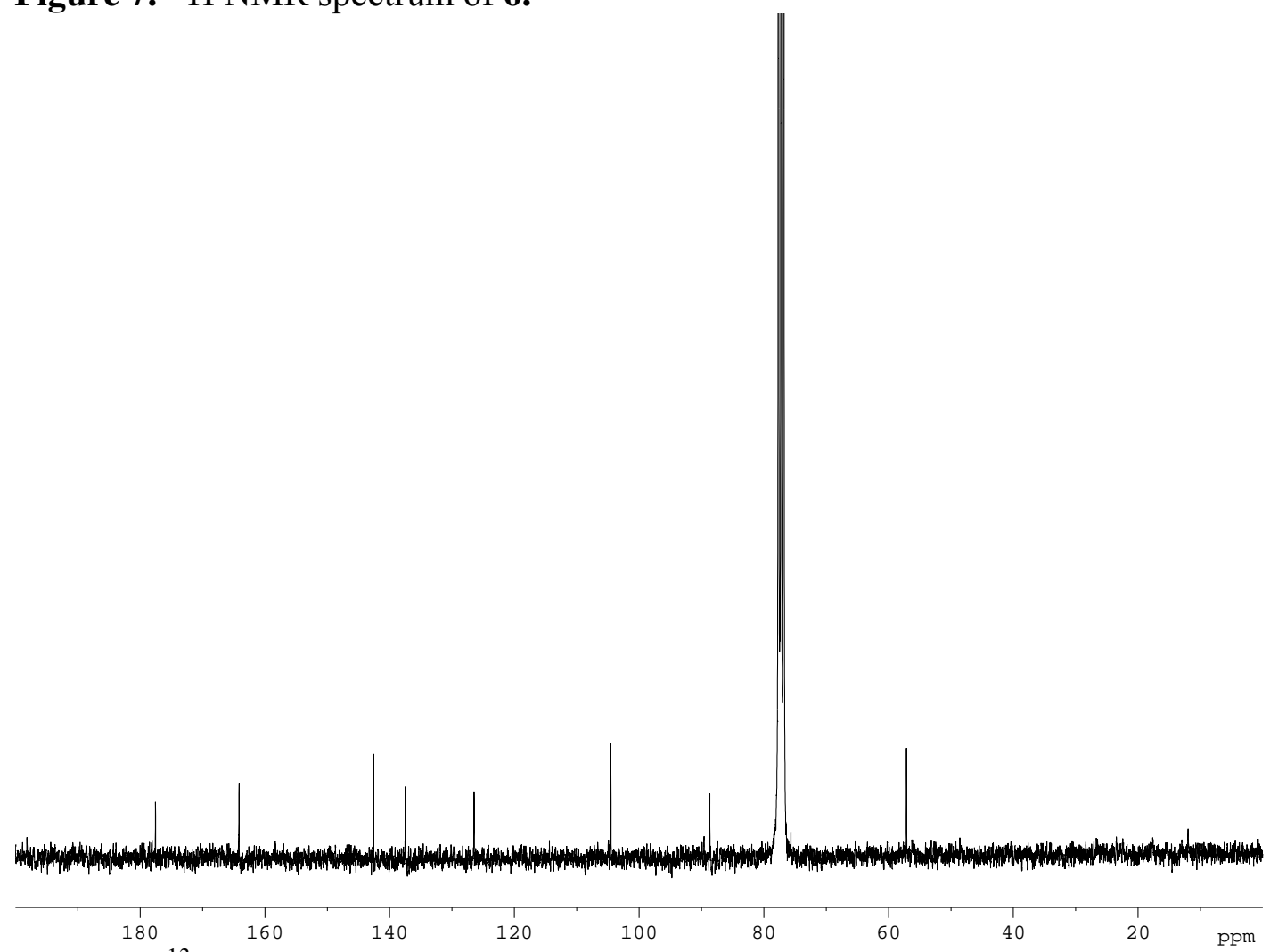

Figure 8. ${ }^{13} \mathrm{C}$ NMR spectrum of 6 . 

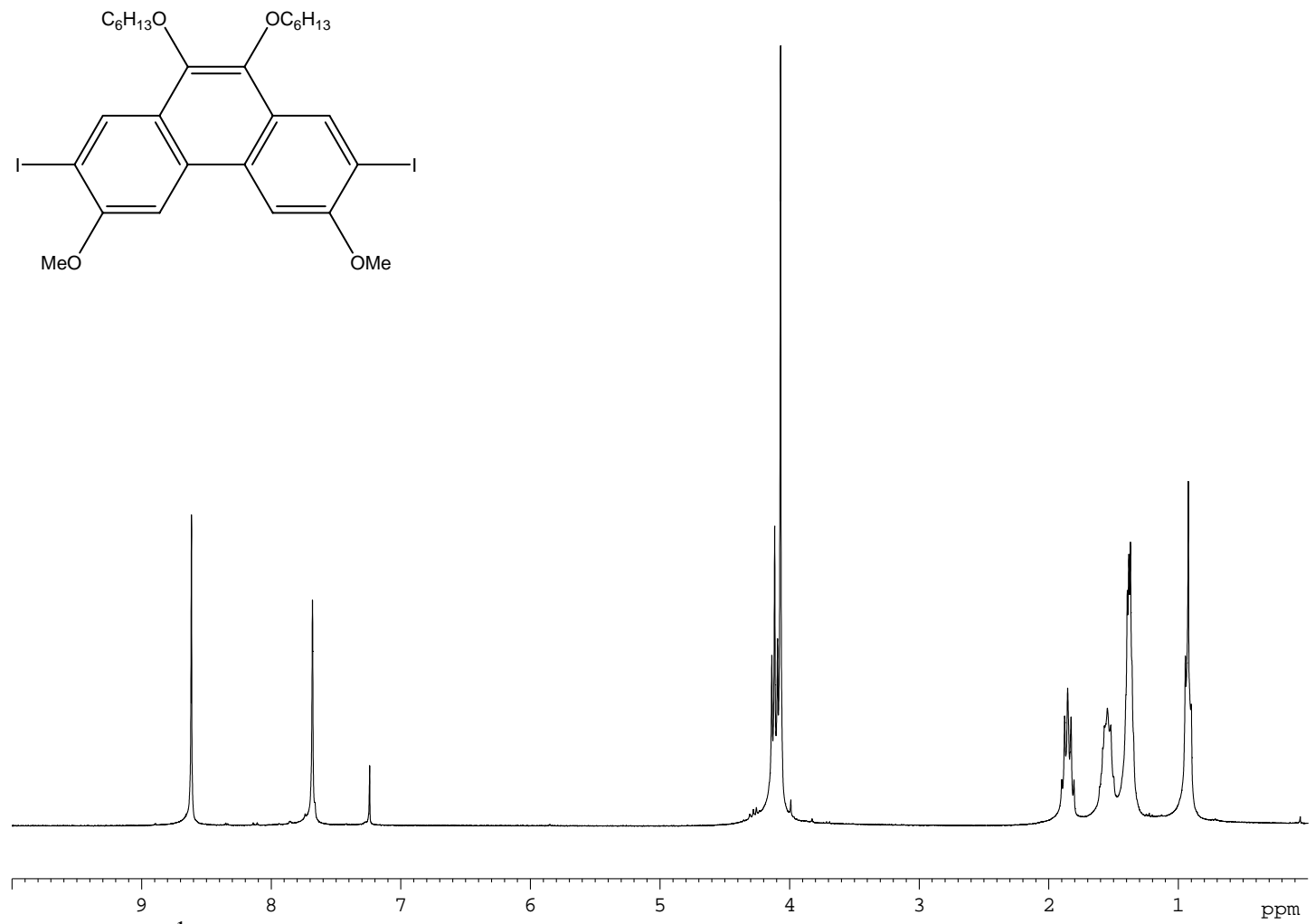

Figure 9. ${ }^{1} \mathrm{H}$ NMR spectrum of 7a.

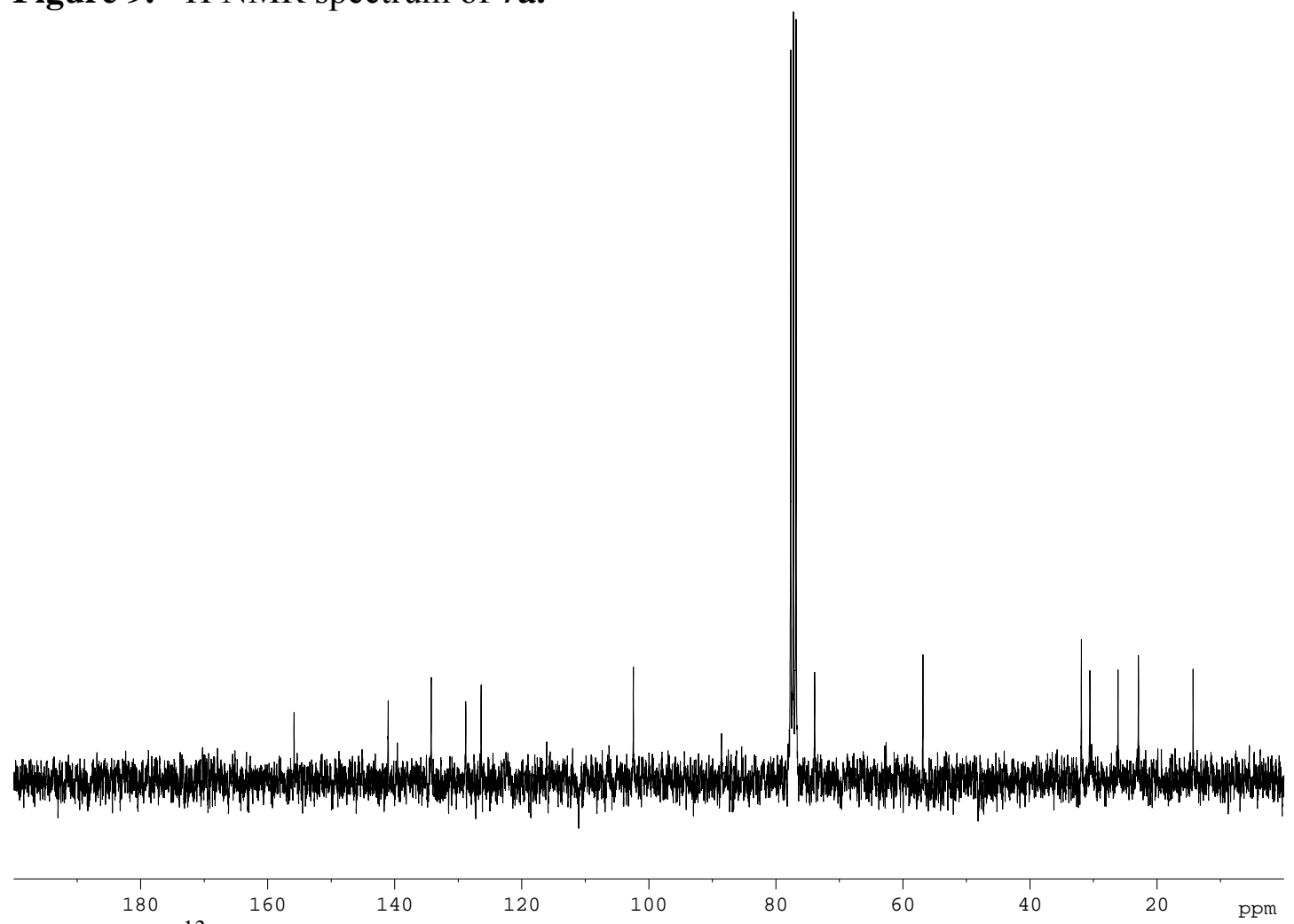

Figure 10. ${ }^{13} \mathrm{C}$ NMR spectrum of 7a. 


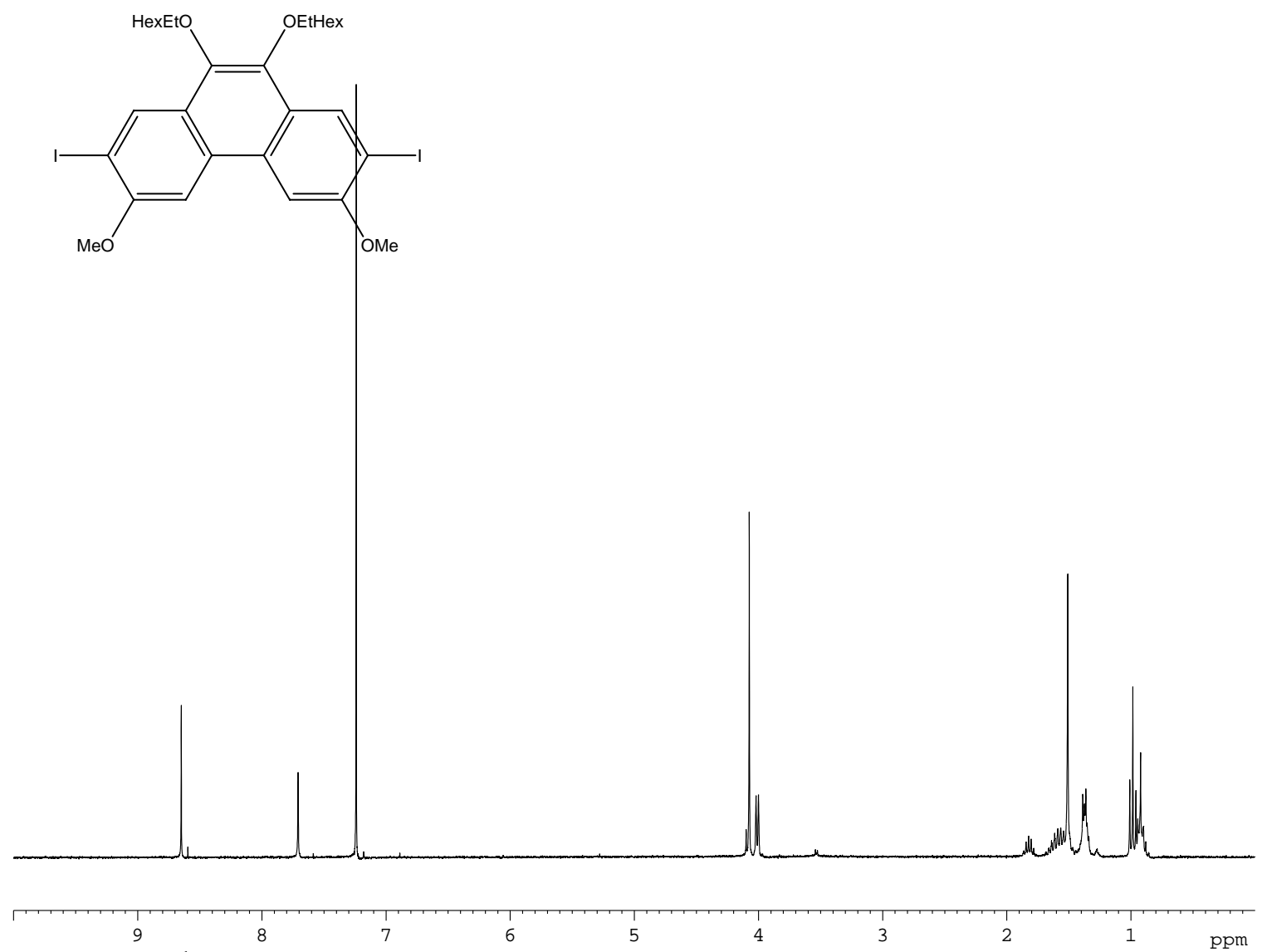

Figure 11. ${ }^{1} \mathrm{H}$ NMR spectrum of $\mathbf{7 b}$.

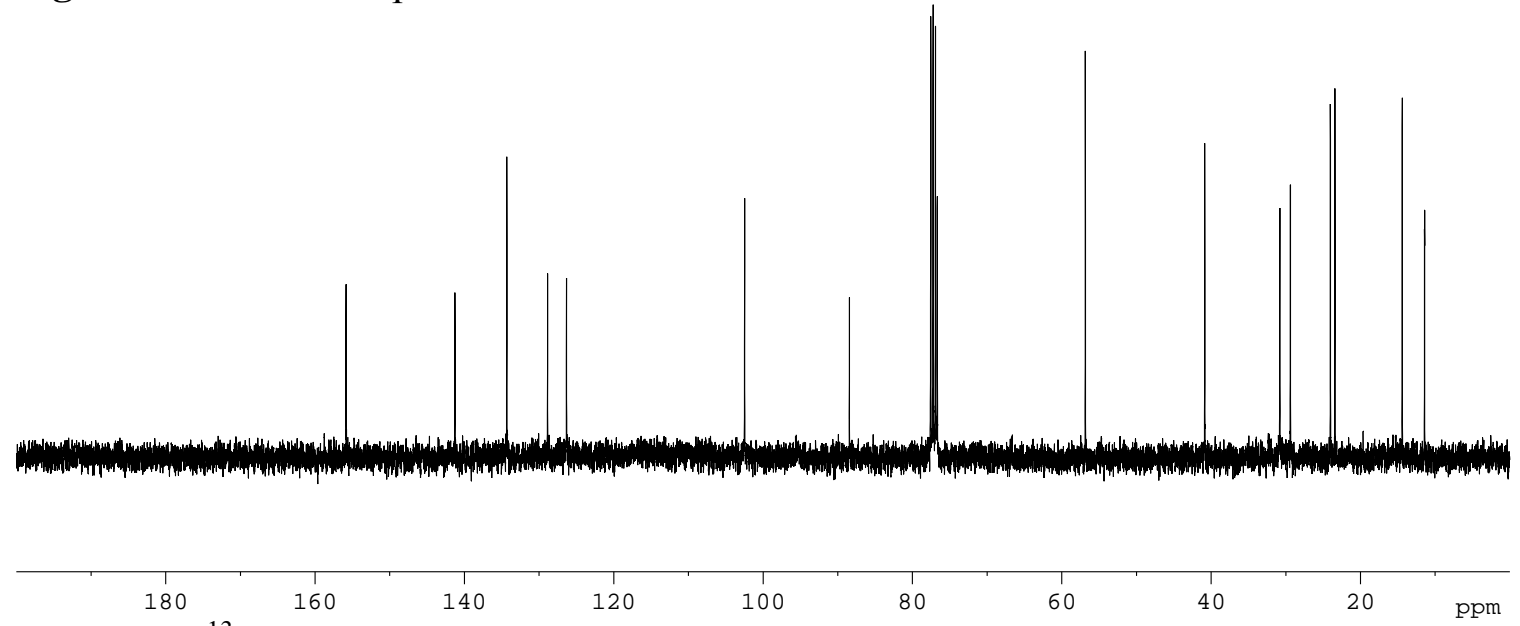

Figure 12. ${ }^{13} \mathrm{C}$ NMR spectrum of $\mathbf{7 b}$. 

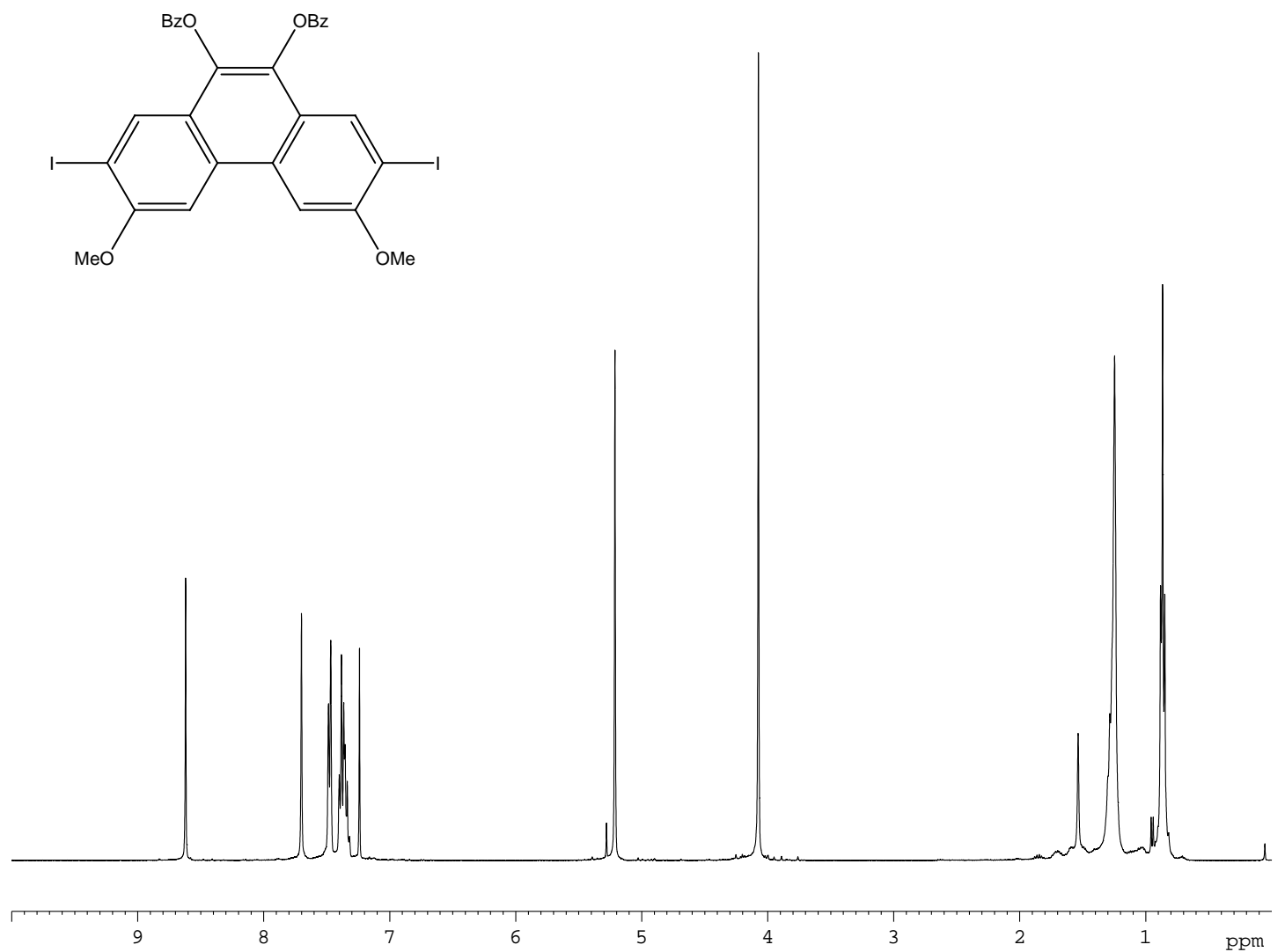

Figure 13. ${ }^{1} \mathrm{H}$ NMR spectrum of 7c.

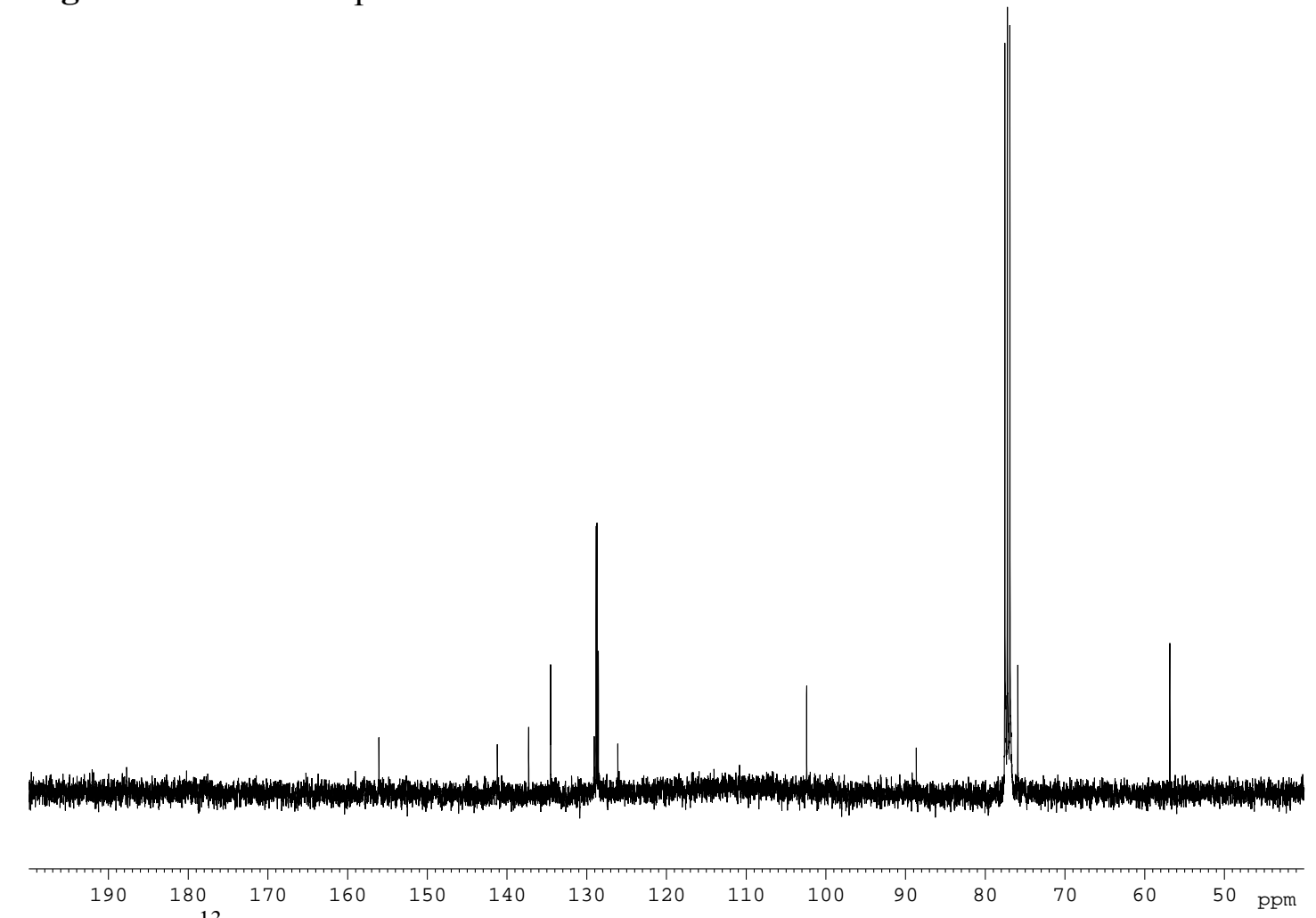

Figure 14. ${ }^{13} \mathrm{C}$ NMR spectrum of 7c. 


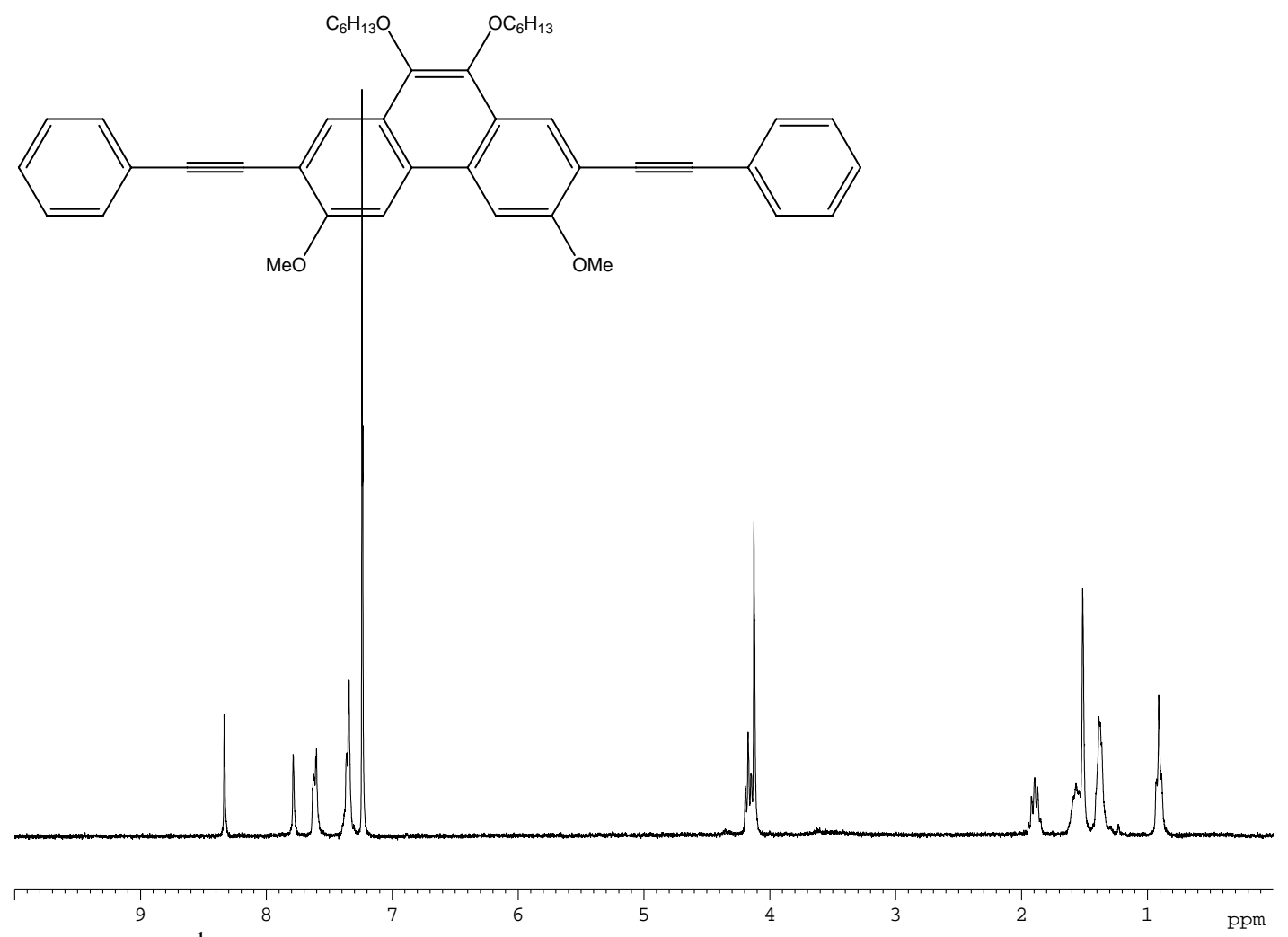

Figure 15. ${ }^{1} \mathrm{H}$ NMR spectrum of 8

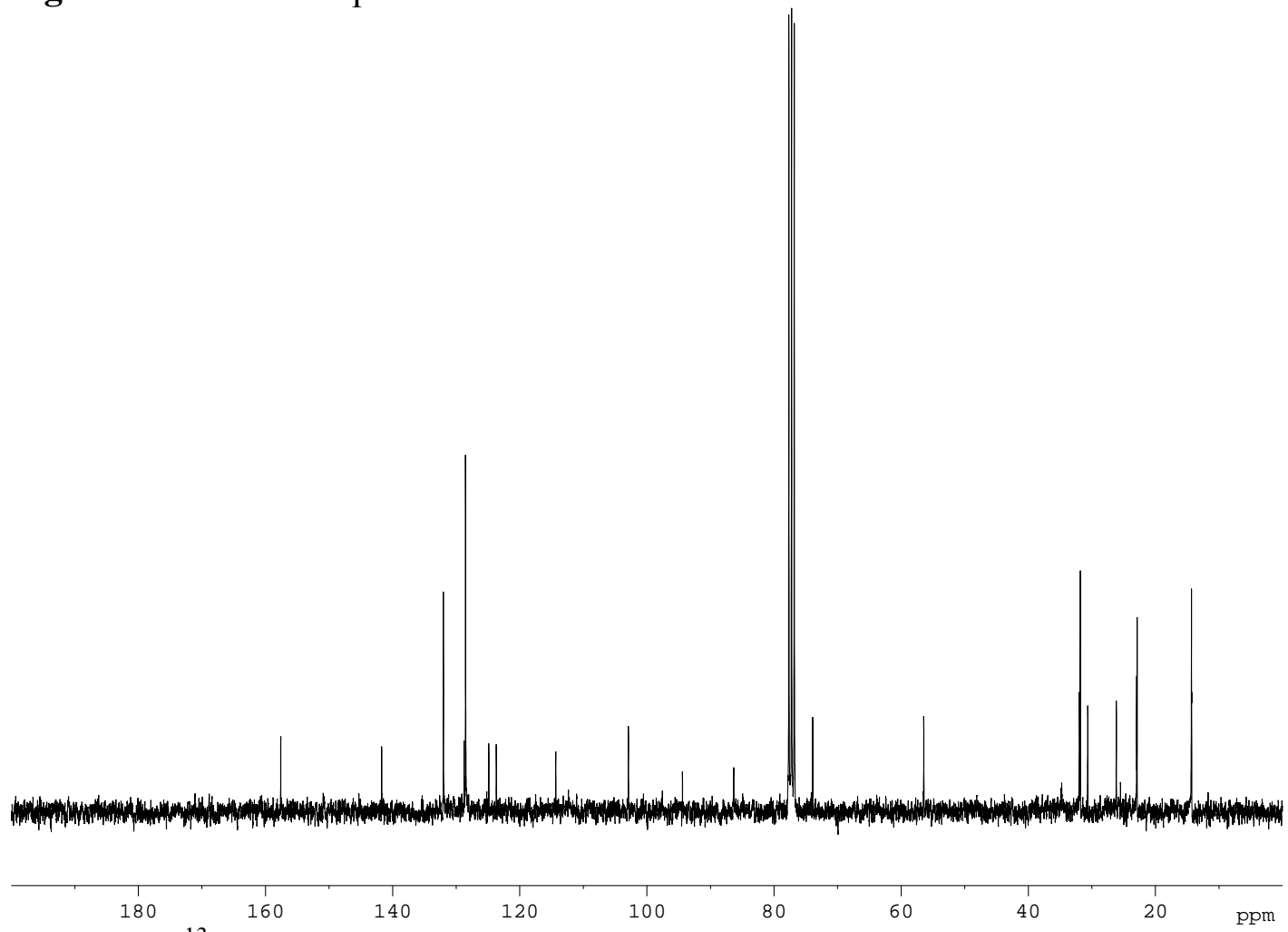

Figure 16. ${ }^{13} \mathrm{C}$ NMR spectrum of 8 . 


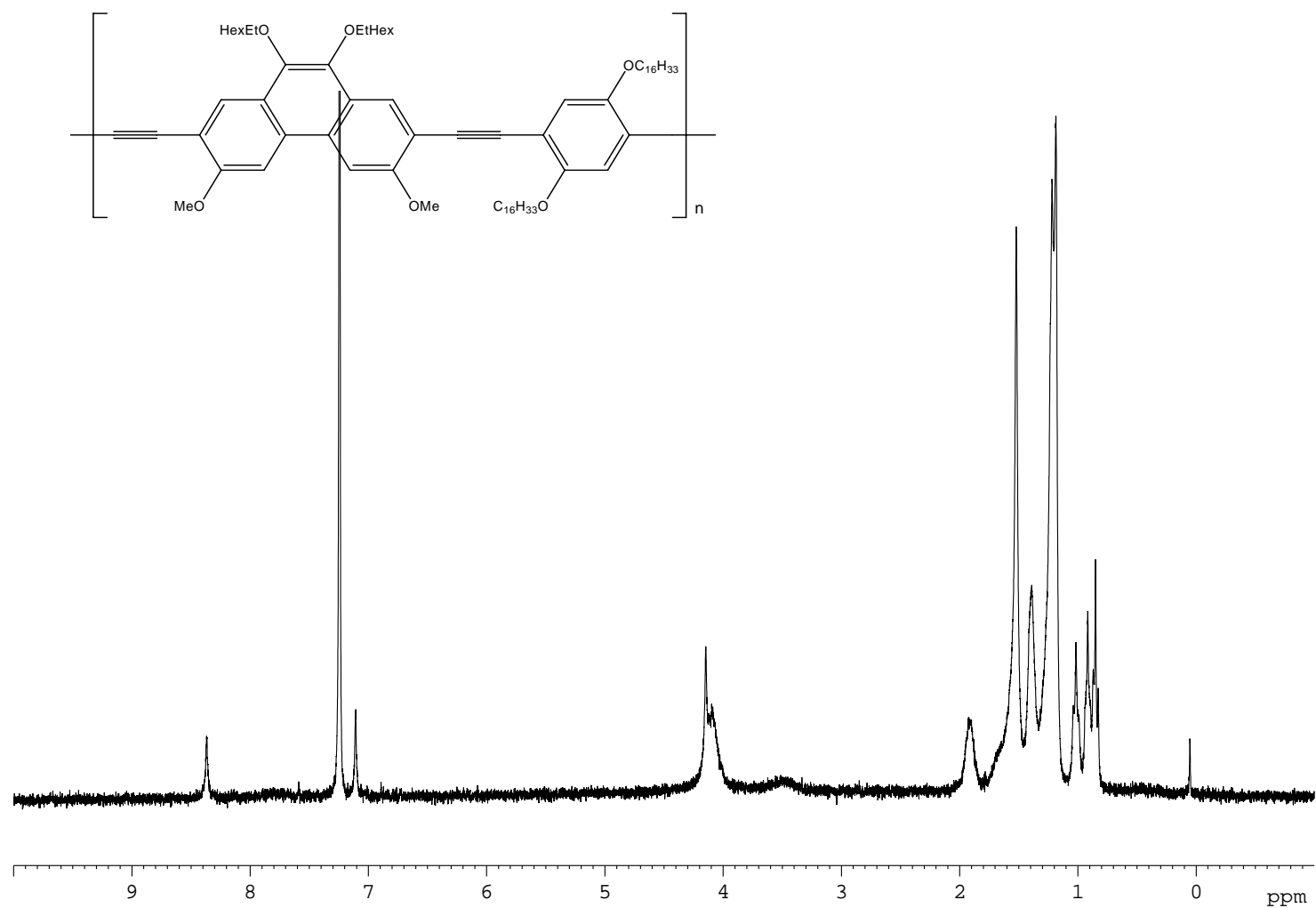

Figure 17. ${ }^{1} \mathrm{H}$ NMR spectrum of $\mathbf{1 0}$.

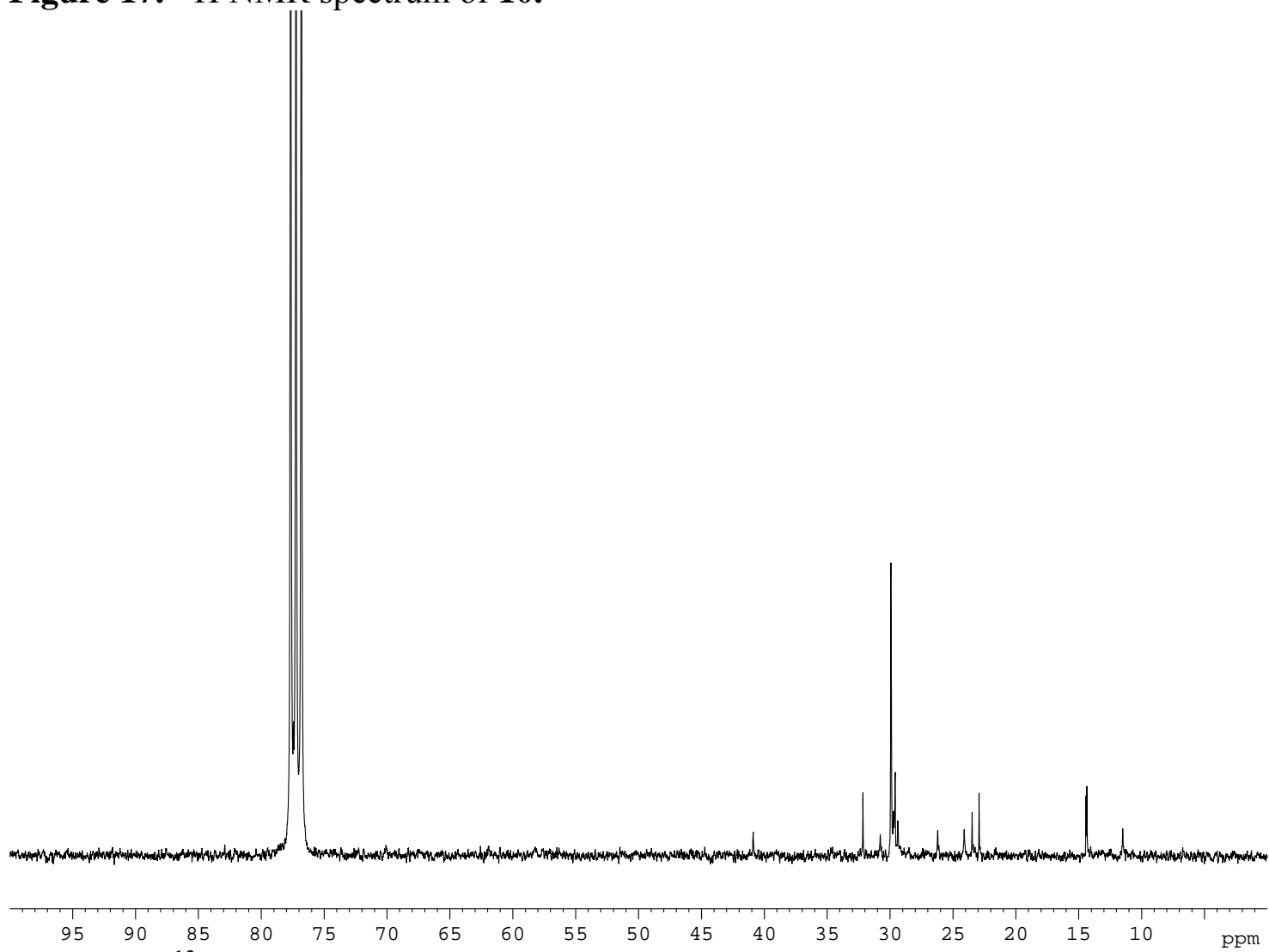

Figure 18. ${ }^{13} \mathrm{C}$ NMR spectrum of $\mathbf{1 0}$. 


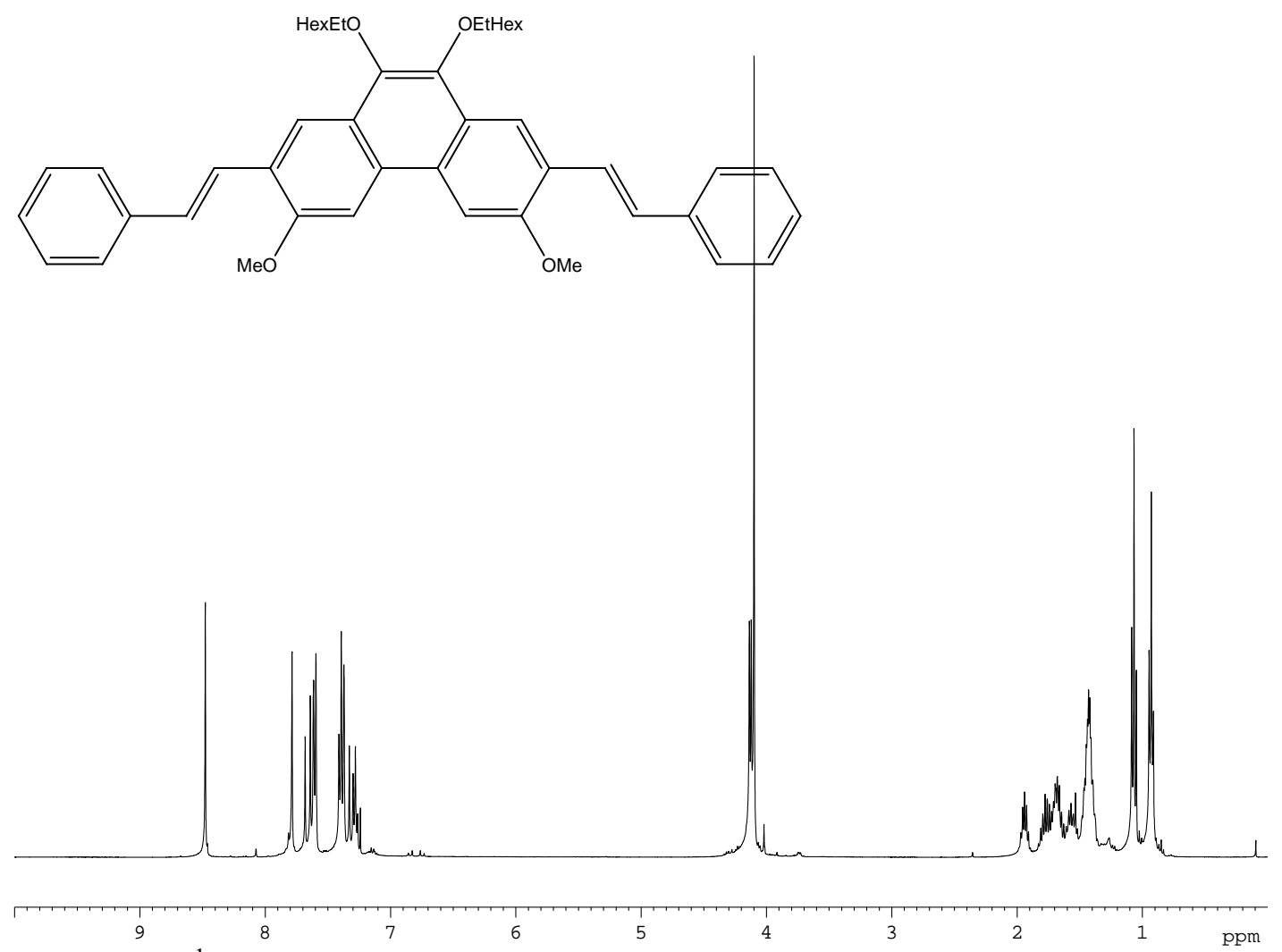

Figure 19. ${ }^{1} \mathrm{H}$ NMR spectrum of 11.

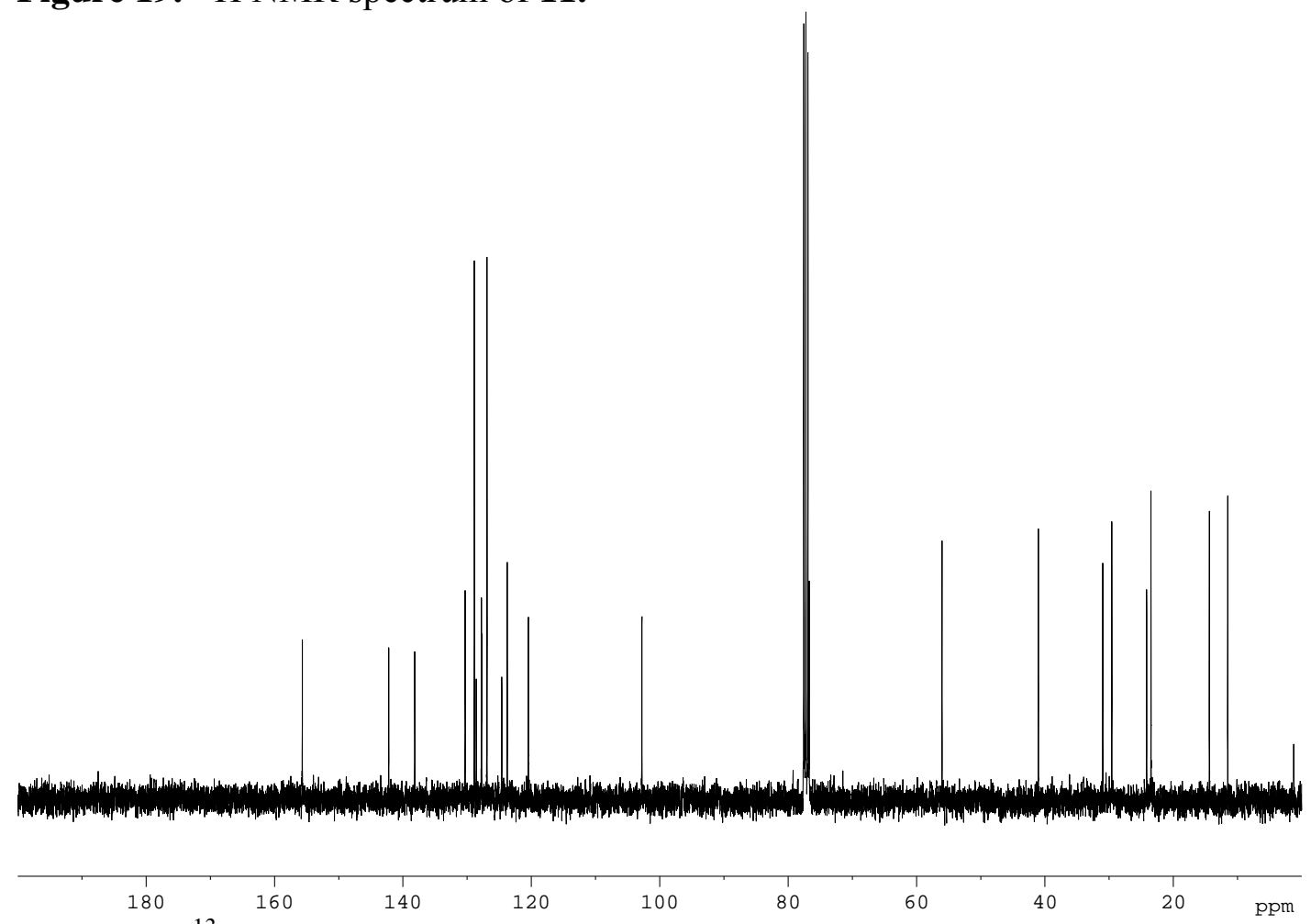

Figure 20. ${ }^{13} \mathrm{C}$ NMR spectrum of 11. 


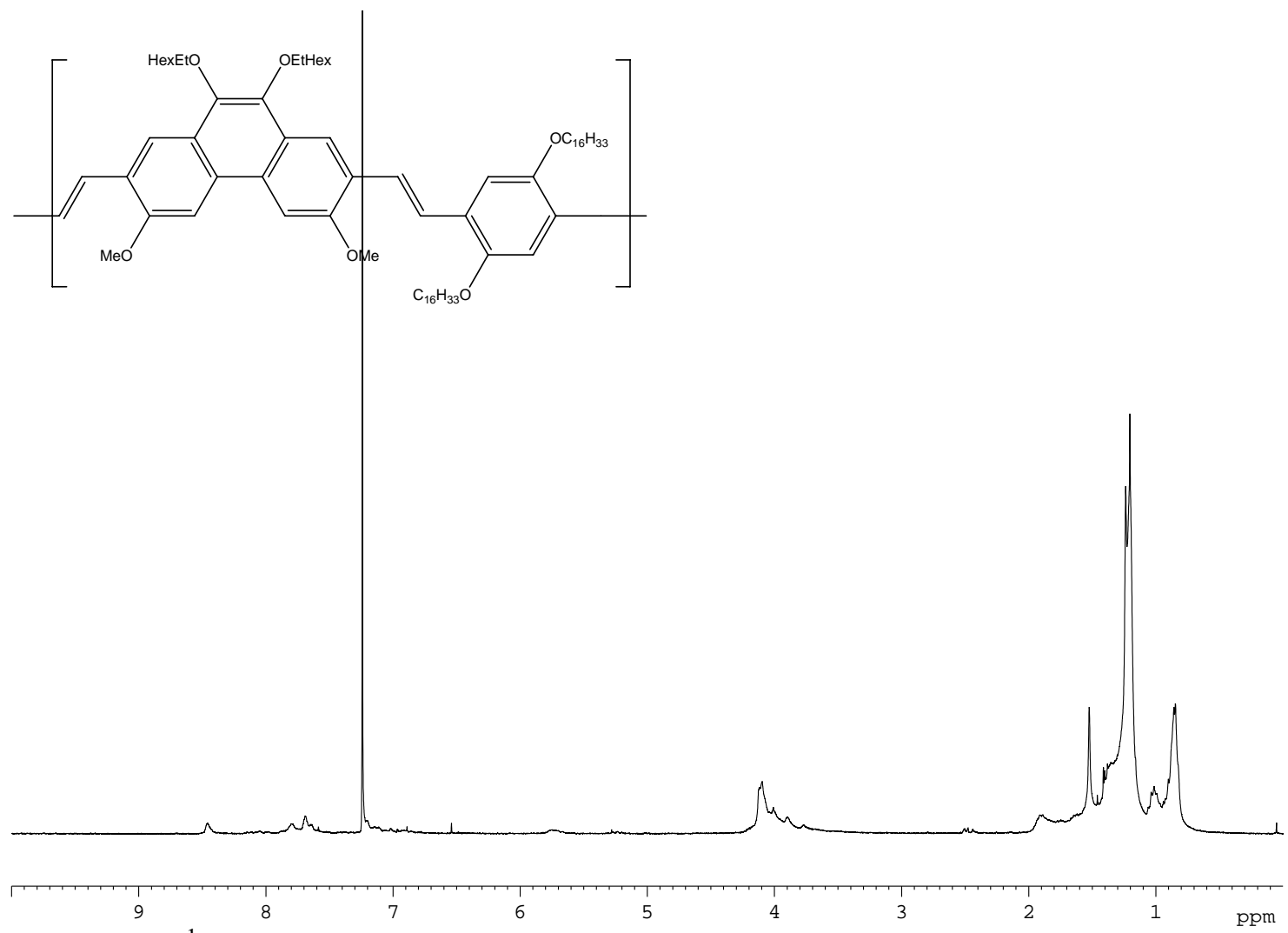

Figure 21. ${ }^{1} \mathrm{H}$ NMR spectrum of 13.
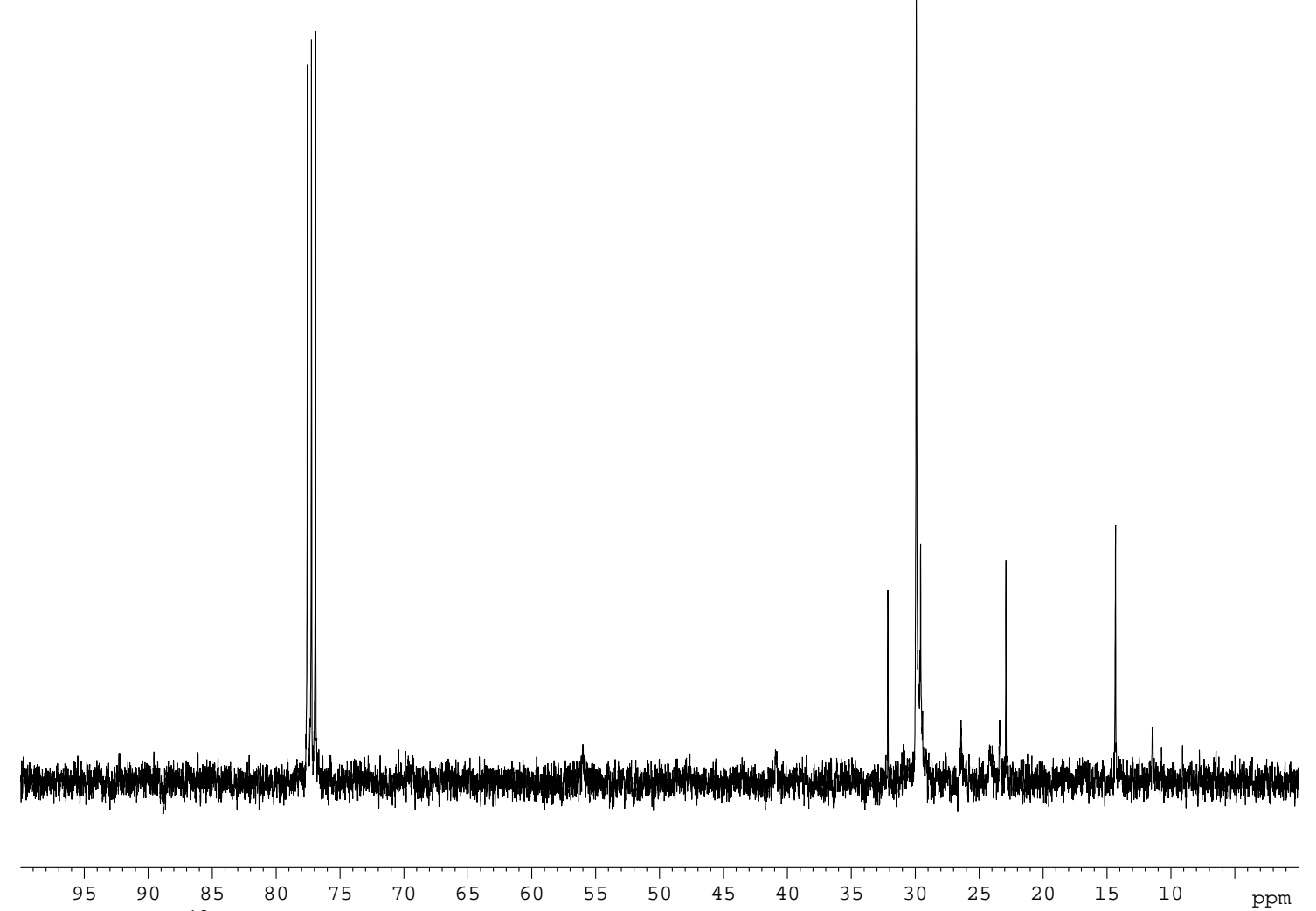

Figure 22. ${ }^{13} \mathrm{C}$ NMR spectrum of 13 . 

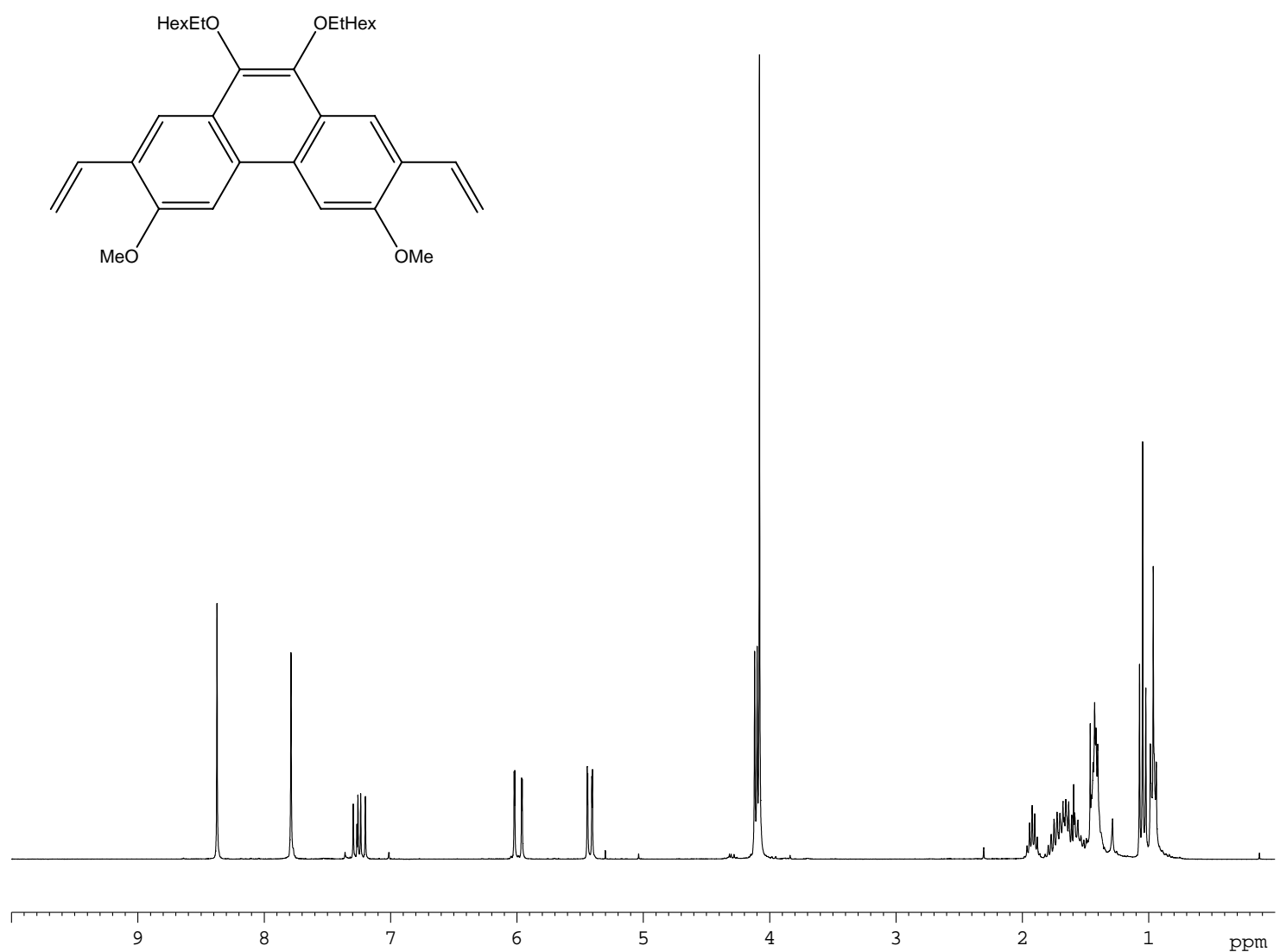

Figure 23. ${ }^{1} \mathrm{H}$ NMR spectrum of 14.

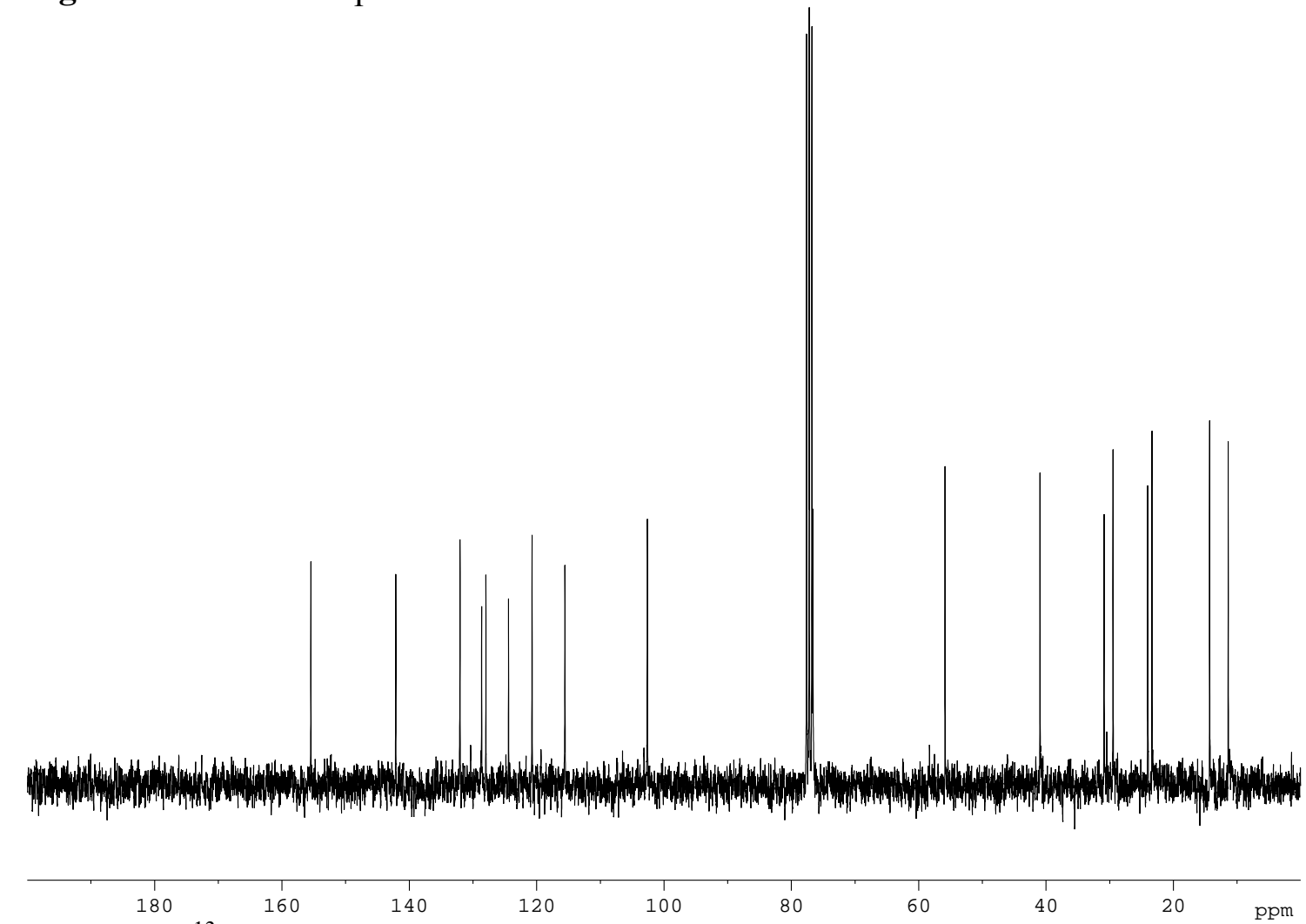

Figure 24. ${ }^{13} \mathrm{C}$ NMR spectrum of 14 . 


\section{References}

${ }^{1}$ Bhatt, M. V. Tetrahedron 1964, 20, 803.

${ }^{2}$ Swager, T. M.; Gil, C. J.; Wrighton, M. S. J. Phys. Chem. 1995, 99, 4886.

${ }^{3}$ Peng, Z.; Galvin, M. E. Chem. Mater.1998, 10, 1785.

${ }^{4}$ Parker, C. A. Photoluminescence of Solutions $1^{\text {rst }}$ Ed.; Elsevier Publishing Company: Amsterdam, 1968; $p$ 261.

${ }^{5}$ Paruch, K.; Katz, T. J.; Incarvito; C.; Lam, K.-C.; Rhatigan, B.; Rheingold, A. L. J. Org. Chem. 2000, 65, 7602. 